\title{
Constraining the primordial magnetic field with dwarf galaxy simulations
}

\author{
Mahsa Sanati ${ }^{1}$, Yves Revaz ${ }^{1}$, Jennifer Schober ${ }^{1}$, Kerstin E. Kunze ${ }^{2}$, and Pascale Jablonka ${ }^{1,3}$ \\ 1 Institute of Physics, Laboratory of Astrophysics, École Polytechnique Fédéale de Lausanne (EPFL), 1290 Sauverny, Switzerland \\ e-mail: mahsa.sanati@epfl.ch \\ 2 Departamento de Física Fundamental, Universidad de Salamanca, Plaza de la Merced s/n, 37008 Salamanca, Spain \\ 3 GEPI, CNRS UMR 8111, Observatoire de Paris, PSL University, 92125 Meudon, Cedex, France
}

Received 8 May 2020 / Accepted 23 July 2020

\begin{abstract}
Using a set of cosmological hydro-dynamical simulations, we constrained the properties of primordial magnetic fields by studying their impact on the formation and evolution of dwarf galaxies. We performed a large set of simulations (8 dark matter only and 72 chemo-hydrodynamical) including primordial magnetic fields through the extra density fluctuations they induce at small length scales $\left(k \geq 10 h \mathrm{Mpc}^{-1}\right)$ in the matter power spectrum. Our sample of dwarfs includes nine systems selected out of the initial $\left(3.4 \mathrm{Mpc} h^{-1}\right)^{3}$ parent box, resimulated from $z=200$ to $z=0$ using a zoom-in technique and including the physics of baryons. We explored a wide variety of primordial magnetic fields with strength $B_{\lambda}$ ranging from 0.05 to $0.50 \mathrm{nG}$ and magnetic energy spectrum slopes $n_{B}$ from -2.9 to -2.1 . Strong magnetic fields characterized by a high amplitude $\left(B_{\lambda}=0.50,0.20 \mathrm{nG}\right.$ with $\left.n_{B}=-2.9\right)$ or by a steep initial power spectrum slope $\left(n_{B}=-2.1,-2.4\right.$, with $\left.B_{\lambda}=0.05 \mathrm{nG}\right)$ induce perturbations on mass scales from $10^{7}$ to $10^{9} M_{\odot}$. In this context emerging galaxies see their star formation rates strongly boosted. They become more luminous and metal rich than their counterparts without primordial magnetic fields. Such strong fields are ruled out by their inability to reproduce the observed scaling relations of dwarf galaxies. They predict that dwarf galaxies are at the origin of an unrealistically early reionization of the Universe and that they also overproduce luminous satellites in the Local Group. Weaker magnetic fields impacting the primordial density field at corresponding masses $\lesssim 10^{6} M_{\odot}$, produce a large number of mini dark matter halos orbiting the dwarfs, however out of reach for current lensing observations. This study allows us, for the first time, to constrain the properties of primordial magnetic fields based on realistic cosmological simulations of dwarf galaxies.
\end{abstract}

Key words. methods: numerical - dark ages, reionization, first stars - galaxies: dwarf - Galaxy: evolution - magnetic fields

\section{Introduction}

Magnetic fields have been observed on all cosmic scales probed so far, from planets and stars (Stevenson 2010; Reiners 2012) to galaxies (Beck 2001; Beck \& Wielebinski 2013) and galaxy clusters (Clarke et al. 2001; Govoni \& Feretti 2004; Vogt \& Enßlin 2005). There ample observational evidence of magnetic fields with strengths ranging from a few to tens of microgauss, coherent on scales up to ten kpc, detected through radio spectropolarimetry in spiral galaxies like M 51 (Fletcher et al. 2011; Beck 2015), and ultraluminous infrared galaxies (ULIRGs; Robishaw et al. 2008; McBride \& Heiles 2013), but also in high redshift galaxies (Bernet et al. 2008; Mao et al. 2017) and in the interstellar and intergalactic medium (Han 2017). However, understanding the origin and strength of the fields is still a challenge for modern astrophysics.

There are various theories proposed to explain the generation of magnetic fields on large scales and their amplification by a dynamo in collapsed objects (Ichiki \& Takahashi 2006; Ryu et al. 2008; Naoz \& Narayan 2013; Schober et al. 2013), or the generation of magnetic fields on small scales (Widrow 2002; Hanayama et al. 2005; Safarzadeh 2018). However, recent observational evidence based on blazer emissions suggests that intergalactic medium voids could host a weak magnetic field $\sim 10^{-16}$ gauss, coherent on Mpc scales (Neronov \& Vovk 2010; Kandus et al. 2011). Such a field is difficult to be explained by turbulence alone in the late universe (Furlanetto \& Loeb 2001; Bertone et al. 2006) and would perhaps favor a primordial origin in the early universe (Subramanian 2016; Kahniashvili et al. 2020; Jedamzik $\&$ Pogosian 2020).

New observational signatures of primordial magnetic fields have recently been obtained from the Lyman- $\alpha$ forest clouds (Pandey \& Sethi 2013), the two-point shear correlation function from gravitational lensing (Pandey \& Sethi 2012), the SunyaevZel'dovich statistics (Tashiro et al. 2009), the cosmic microwave background (CMB) anisotropies (Shaw \& Lewis 2010; Planck Collaboration Int. XXVI 2015; Zucca et al. 2017; Paoletti et al. 2019), CMB spectral distortions (Jedamzik et al. 2000; Kunze \& Komatsu 2014; Wagstaff \& Banerjee 2015) large-scale structure (Kahniashvili et al. 2010), and the reionization history of of the Universe (Pandey et al. 2015). The above observations provide new upper limits on the strength of the fields which appeared to be limited to $47 \mathrm{pG}$ for scale-invariant fields (Jedamzik \& Saveliev 2019) and a few $\mathrm{nG}$ in more general states coherent on $1 \mathrm{Mpc}$. It is thus of great interest to ask whether such primordial fields can be confirmed and how their characteristics can be constrained further.

To answer this issue we need to journey back to the early universe. The present-day large-scale structures are thought to be seeded by quantum field fluctuations when the relevant scales were causally connected, leading to a nearly scale-invariant fluctuation spectrum (Dodelson 2003; Kolb \& Turner 1990; 
Padmanabhan 2002). These scales then crossed the universe horizon during the inflationary expansion phase, and re-entered only later to serve as the initial conditions, leading to the growth of large-scale structures. It is likely that the origin of the magnetic fields also arose during various phase transitions in the early universe (Turner \& Widrow 1988a; Adshead et al. 2016; Domcke et al. 2019; Fujita \& Durrer 2019) by a small fraction of the energy released during the electroweak or quark-hadron transitions and converted to large-scale magnetic fields (Hogan 1983; Ratra 1992a).

A primordial magnetic field (PMF) in the postrecombination epoch generates density fluctuations in addition to the standard inflationary fluctuations. The magnetically induced perturbations for a scale-invariant magnetic spectrum may dominate the standard Lambda cold dark matter $(\Lambda \mathrm{CDM})$ matter power spectrum on small length scales and therefore can affect the formation of the first galaxies (Kim et al. 1996; Wasserman 1978b). The $\Lambda$ CDM paradigm implies a hierarchical formation of galaxies in which dwarf galaxies, including the ultrafaint dwarfs (UFDs; Simon 2019), as observed today are the best analogs to the smallest initial building blocks. A modification of the nearly power-law spectrum of the $\Lambda \mathrm{CDM}$ model on small scales $\left(k \geq 10 \mathrm{~h} \mathrm{Mpc}^{-1}\right)$ will thus directly affect the number and properties of dwarf galaxies with interesting outcomes for understanding the cosmological model and origin of the magnetic field.

Changing the abundance of dwarf galaxies and their structure can potentially shed new light on the long-standing tensions existing between dark matter only (DMO) $\Lambda$ CDM cosmological simulations and Local Group observations (see Bullock \& Boylan-Kolchin (2017) for a recent review). Among the existing tensions, the overprediction of low mass systems around the Milky Way, the so-called missing satellite problem (Klypin et al. 1999; Moore et al. 1999), and the too-big-to-fail problem (Boylan-Kolchin et al. 2011, 2012) are certainly the most emblematic. Along with the number of observed satellites, their mass profile is also known to suffer from tensions. DMO simulations predict that the dark matter halos follow a universal cuspy profile (Navarro et al. 1996, 1997), while observations favor cored ones (Moore 1994; Flores \& Primack 1994, see Read et al. 2018 for a short review). While improvements in the baryonic treatment of cosmological simulations allowed for a reduction of those tensions (Chan et al. 2015; Sawala et al. 2016), (Wetzel et al. 2016; Verbeke et al. 2017; Revaz \& Jablonka 2018; Pontzen \& Governato 2014; Oñorbe et al. 2015; Chan et al. 2015; Fitts et al. 2017; Hausammann et al. 2019), modification or alternative to the $\Lambda \mathrm{CDM}$ have also been proposed, such as, like warm dark matter (Lovell et al. 2012; Governato et al. 2015; Fitts et al. 2019), self-interacting dark matter (Vogelsberger et al. 2014; Harvey et al. 2018; Fitts et al. 2019) or wave dark matter (Chan et al. 2018).

On the other hand, many observations available for dwarf galaxies in the Local Group may be used to constrain the amplitude of PMFs. Any model including the effect of PMFs must (i) reproduce the abundances of detected satellites around the Milky Way (Newton et al. 2018; Nadler et al. 2018; DrlicaWagner et al. 2020); (ii) reproduce the well-known observed scaling relations (Mateo 1998; Tolstoy et al. 2009; McConnachie 2012; Simon 2019), as well as more detailed properties like lineof-sight (LOS) velocity dispersion, stellar abundance patterns, and star formation histories (Tolstoy et al. 2009); and (iii) must be in agreement with the Milky Way local reionization history. By hosting the first generation of stars and lightening up the dark ages (Choudhury et al. 2008; Salvadori et al. 2014; Wise et al. 2014; Robertson et al. 2015; Bouwens et al. 2015; Atek et al. 2015), dwarf galaxies played a key role during the epoch of reionization.

The above arguments make dwarf and UFD galaxies excellent laboratories to study the subtle impact of initial conditions imposed after the inflation and how they rule the formation of first structures. As such, they may be used to get new constraints on the properties of PMFs (Safarzadeh \& Loeb 2019).

Revaz \& Jablonka (2018) have recently demonstrated that cosmological simulations performed with the code GEAR reproduce a wide range of observed properties of the Local Group dwarf galaxies. Relying on this previous work, we take the opportunity to study for the first time the formation and evolution of a suite of dwarf galaxies that emerge from a $\Lambda \mathrm{CDM}$ cosmology where the primordial density fluctuations include the effect of PMFs. Our goal is to determine the impact of these primordial fields on the properties of dwarf galaxies.

This paper is organized as follows. Section 2 recalls the theory around PMFs and their effect on the density fluctuation power spectrum. Section 3 describes in detail our numerical framework as well as the simulations performed. The results are presented in Sect. 4. We first discuss results relying on dark matter only simulations and show how the PMFs affect the matter halo mass function on small scales. We then focus on zoom-in hydo-dynamical simulations. We study the impact of PMFs on the properties of dwarf galaxies and in particular on scaling relations. We then estimate how the modified properties of dwarfs directly impact the reionization history of the Universe as well as the cumulative number of bright satellites around the Milky Way. Limits on the properties of PMFs are obtained by comparing with existing observational constraints. A brief conclusion is given in Sect. 5.

\section{Impact of primordial magnetic fields on the density fluctuations}

We consider the effect of PMFs generated before recombination by processes occurring during the inflationary epoch (e.g., Turner \& Widrow 1988b; Ratra 1992b). It has been shown in the seminal works of Wasserman (1978a) and Kim et al. (1996) that after the recombination epoch, PMFs may induce motions in the ionized baryons through the Lorentz force which drives compressional and rotational perturbations causing density fluctuations in the gas. These perturbations propagate down to the neutral gas and also to the dark matter through the gravitational coupling, and a direct impact on the total matter power spectrum is expected.

Characterizing this impact, however, is non-trivial and a large literature has been dedicated to describe it. Hereafter, without entering into too much detail, we briefly review the principal steps that allow us to determine the origin of the effect of primordial magnetic fields on the total matter power spectrum.

\subsection{Primordial magnetic field}

Following Wasserman (1978a), Kim et al. (1996), and Gopal \& Sethi (2003), we first assume that the tangled magnetic field results from a statistically homogeneous and isotropic vector random process. The two-point correlation function of a nonhelical field in Fourier space can then be expressed as

$\left\langle\hat{B}_{i}(\boldsymbol{k}) \hat{B}_{j}^{*}\left(\boldsymbol{k}^{\prime}\right)\right\rangle=(2 \pi)^{3} \delta\left(\boldsymbol{k}-\boldsymbol{k}^{\prime}\right) \frac{P_{i j}\left(\boldsymbol{k}, \boldsymbol{k}^{\prime}\right)}{2} P_{B}(k)$, 
where $P_{i j}(\boldsymbol{k})=\delta_{i j}-\frac{k_{i} k_{j}}{k^{2}}, k=|\boldsymbol{k}|$ is the comoving wave number and $P_{B}(k)$ is the magnetic field power spectrum. Without further information on the exact origin of the PMFs, it is usually assumed that its power spectrum follows a simple power law

$P_{B}(k)=A k^{n_{B}}$

where $n_{B}$ is the slope index and $A$ is the amplitude of the magnetic spectrum, which is defined through the variance of the magnetic field strength $B_{\lambda}^{2}$ on a scale $\lambda=1 \mathrm{Mpc} \equiv k_{\lambda}^{-1}$, namely (Shaw \& Lewis 2012):

$A=\frac{(2 \pi)^{n_{B}+5} B_{\lambda}^{2}}{2 \Gamma\left(\frac{n_{B}+3}{2}\right) k_{\lambda}^{n_{B}+3}}$.

With these definitions, $n_{B}$ and $B_{\lambda}$ fully characterize the PMFs. They constitute our main free parameters.

\subsection{Growth of perturbations}

In the linearized Newtonian theory, the evolution of density fluctuations in the presence of PMFs is described by the two following coupled equations for the baryonic fluid perturbation field $\delta_{b}(\boldsymbol{x}, t)=\delta \rho_{b}(\boldsymbol{x}, t) / \bar{\rho}_{b}$ and for the collisionless dark matter $\delta_{\mathrm{DM}}(\boldsymbol{x}, t)=\delta \rho_{\mathrm{DM}}(\boldsymbol{x}, t) / \bar{\rho}_{\mathrm{DM}}$ (see, e.g., Subramanian $\&$ Barrow 1998; Sethi \& Subramanian 2005):

$$
\begin{aligned}
\frac{\partial^{2}}{\partial t^{2}} \delta_{b}+\left[2 H+\frac{4 \rho_{\gamma}}{3 \rho_{b}} n_{e} \sigma_{T} a\right] \frac{\partial}{\partial t} \delta_{b} & =c_{b}^{2} \nabla^{2} \delta_{b}+4 \pi G a^{2} \\
& \times\left[\rho_{b} \delta_{b}+\rho_{\mathrm{DM}} \delta_{\mathrm{DM}}\right]+\frac{S(\boldsymbol{x}, t)}{a^{3}}
\end{aligned}
$$

and

$$
\frac{\partial^{2}}{\partial t^{2}} \delta_{\mathrm{DM}}+2 H \frac{\partial}{\partial t} \delta_{\mathrm{DM}}=4 \pi G a^{2}\left[\rho_{b} \delta_{b}+\rho_{\mathrm{DM}} \delta_{\mathrm{DM}}\right]
$$

Here $a$ is the scale factor, $H=\dot{a} / a$ the Hubble constant, $\rho_{\mathrm{b}}$ and $\rho_{\gamma}$ the baryon and photon mass density, $n_{\mathrm{e}}$ the electron number density, $\sigma_{\mathrm{T}}$ the Thomson cross section for electron-photon scattering, and $c_{\mathrm{b}}$ the baryon sound speed. The magnetic field source term, $S$, represents normalized to the baryon density at the present time, $\bar{\rho}_{\mathrm{b}}\left(t_{0}\right)$ :

$S(\boldsymbol{x}, t)=\frac{\boldsymbol{\nabla} \cdot[\boldsymbol{B} \times(\boldsymbol{\nabla} \times \boldsymbol{B})]}{4 \pi \bar{\rho}_{\mathrm{b}}\left(t_{0}\right)}$.

The baryon pressure term $c_{\mathrm{b}}^{2} \nabla^{2} \delta_{b}$ in Eq. (4) is sub-dominant with respect to the magnetic pressure as long as the background magnetic field is larger than $5 \times 10^{-11} \mathrm{G}$ (Subramanian $\&$ Barrow 1998) and may thus be ignored. The damping term involving the Thomson cross section corresponds to the radiation viscosity. Prior to recombination, this term leads to the damping of small-scale magnetic waves (Jedamzik et al. 1998; Subramanian \& Barrow 1998), a physical process similar to Silk damping (Silk 1968). This induces a sharp cutoff of the magnetic field when entering the recombination epoch, and subsequently of its contribution in the total matter power spectrum.

We introduce the total matter density perturbation:

$$
\delta_{m}(\boldsymbol{x}, t)=\left(\bar{\rho}_{\mathrm{DM}} \delta_{\mathrm{DM}}+\bar{\rho}_{\mathrm{b}} \delta_{\mathrm{b}}\right) /\left(\bar{\rho}_{\mathrm{DM}}+\bar{\rho}_{\mathrm{b}}\right) .
$$

We can then solve Eqs. (4) and (5) and get the time evolution of $\delta_{m}$. The additional term in the evolution of $\delta_{m}$, due only to the presence of the magnetic fields, is found to be (Sethi \& Subramanian 2005):

$\delta_{m} \sim \frac{3}{5} \frac{\Omega_{\mathrm{b}}}{\Omega_{\mathrm{m}}^{2}}\left[\frac{3}{2}\left(\frac{t}{t_{\mathrm{rec}}}\right)^{2 / 3}+\left(\frac{t_{\mathrm{rec}}}{t}\right)-\frac{5}{2}\right] S\left(\boldsymbol{x}, t_{\mathrm{rec}}\right) t_{\mathrm{rec}}^{2}$,

where $t_{\mathrm{rec}}$ is the time at recombination and $\Omega_{\mathrm{m}}$ and $\Omega_{\mathrm{b}}$ are the matter and baryon density parameters.

The important point to get from the previous equations is that the spatial dependence of $\delta_{m}(\boldsymbol{x}, t)$ can be followed through the magnetic field source term $S\left(\boldsymbol{x}, t_{\mathrm{rec}}\right)$. We thus expect the total matter power spectrum to directly depend on the power spectrum of the magnetic field.

It is worth noting that the most significant evolution of the magnetic field takes place before recombination (Kahniashvili \& Tevzadze 2013a; Brandenburg et al. 2017a,b). During recombination the ionization degree drops to a tiny value. Afterward, the magnetic field is more or less frozen into the gas, i.e., it just passively follows the cosmic expansion, ensuring that the magnetic flux is conserved.

\subsection{Impact on the total matter power spectrum}

The modification of the total matter power spectrum due to the influence of magnetic fields was first addressed by Kim et al. (1996) and was extended by Gopal \& Sethi (2003). The total matter power spectrum is the ensemble average of the density fluctuations in Fourier space, $P(k, t)=\left\langle\delta_{\mathrm{m}}(k, t) \delta_{m}^{*}(k, t)\right\rangle$, that we can obtain from the evolution equation (Eq. (7)) and introducing the ensemble average of the PMFs, Eqs. (6) and (2).

After the decoupling of photons, the ionized matter density fluctuations are affected by the magnetic Alfvén waves if the crossing time, $\tau_{A} \sim 1 / k v_{A}$, is smaller than the inverse Hubble rate (Banerjee \& Jedamzik 2004), where $v_{\mathrm{A}}$ is the Alfvén velocity $v_{\mathrm{A}}=B_{\lambda} / \sqrt{\mu_{0} \rho_{0}}$, with $\mu_{0}$ the permeability or magnetic constant. As the magnetic energy $B_{\lambda}^{2}$ scales with $P_{B}(k) k^{3}, \tau_{\mathrm{A}} \sim$ $\sqrt{\mu_{0} \rho_{0}} k^{-\left(3+n_{B}\right) / 2}$. For $n_{B}>-5$, the crossing time is then shorter for larger $k$, amplifying the perturbations faster on smaller scales. In the limit where $k \ll k_{B}$, with $k_{B} \cong\left(v_{\mathrm{A}} \sqrt{\pi / \rho_{0} G}\right)^{-1}$, the magnetic wave number above the Alfvén waves damp instabilities (the equivalent of the Jeans wave number), at the lowest order in $k / k_{\text {max }}$ (i.e., $k_{\max } \cong k_{B}$ ), the solution for the total matter power spectrum is (Gopal \& Sethi 2003)

$P(k) \sim A k^{2 n_{B}+7}+B k_{\max }^{2 n_{B}+3} k^{4}+C k_{\max }^{2 n_{B}+1} k^{6}+\ldots$,

which strongly depends on the magnetic slope index $n_{B}$. For the scale-invariant case, $n_{B} \cong-3, P(k) \sim k$, and $\tau_{A} \sim k^{-1}$, thus small-scale perturbations are strongly amplified, as long as $\tau_{A}<$ $1 / H(t)$, up to about $k_{B}$ where they are sharply quenched (see Sect. 2.2).

\subsection{Adopted total matter power spectrum}

We generated the matter power spectrum using a modified version of the CAMB code which includes the effects of PMFs (for details see Shaw \& Lewis 2012). In this version the non-linear effects of the magnetic Jeans length (magnetic pressure) and the damping due to the radiation viscosity (when the photon free streaming length is small) are both explicitly computed. As such, contrary to other studies, there is no need to artificially include a cutoff wave number.

Figures 1 and 2 display the matter power spectrum due to the magnetic field (dashed lines) together with its contribution to 


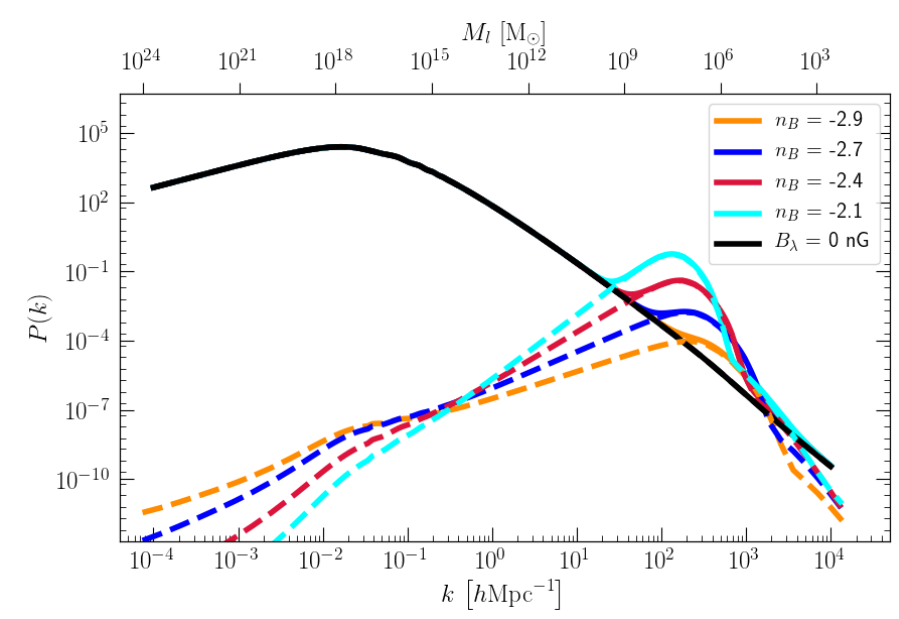

Fig. 1. Contribution of the magnetically induced power spectrum for a variety of magnetic field indexes (dashed colored lines) to the total matter power spectrum solid colored lines). The unperturbed $\Lambda \mathrm{CDM}$ spectrum is shown as the black continuous line. The magnetic field strength is kept constant at $B_{\lambda}=0.05 \mathrm{nG}$.

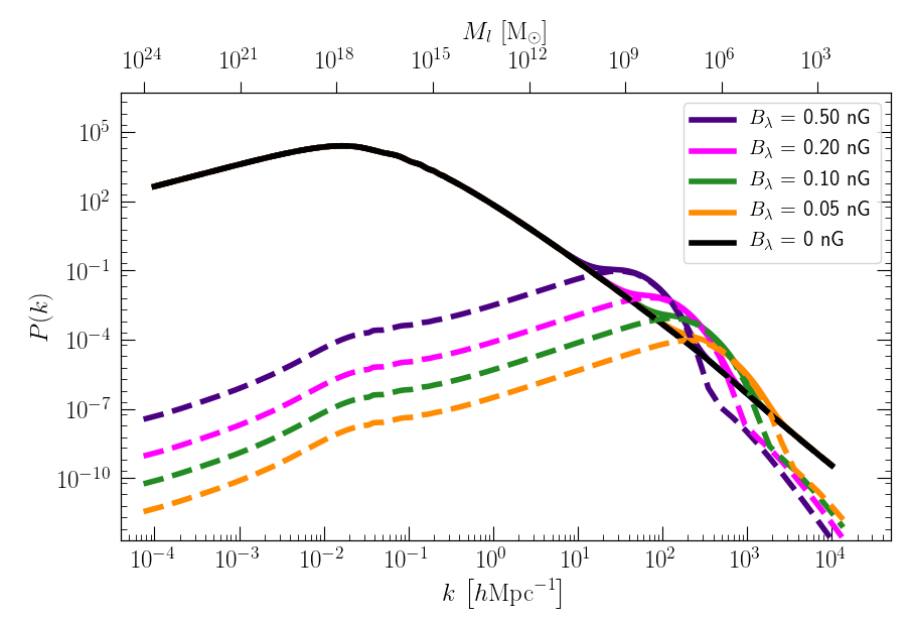

Fig. 2. Contribution of the magnetically induced power spectrum to the total matter power spectrum. Same as Fig. 1, but with constant spectral slope, $n_{B}=-2.9$, and varying magnetic field amplitudes.

the total matter power spectrum (plain lines). The black continuous line corresponds to the unperturbed $\Lambda$ CDM power spectrum. The dependence with respect to the magnetic field amplitude $\left(B_{\lambda}=0.05,0.10,0.20,0.50 \mathrm{nG}\right)$ is shown in Fig. 1 keeping the spectral index constant $\left(n_{B}=-2.9\right)$. The dependence with respect to the spectral index with $B_{\lambda}=0.05 \mathrm{nG}$ is shown in Fig. 2. The range of parameters explored is chosen in such a way that they impact the power spectrum without violating the existing constraints, as do those presented in Sect. 1.

It is worth mentioning, for the magnetic spectral index, that a range of both positive and negative values are suggested. In this study, we considered PMFs generated during the inflation, which tend to have negative values for $n_{B}$, (Turner \& Widrow 1988a; Ratra 1992a; Giovannini \& Shaposhnikov 2000, Martin \& Yokoyama 2008). However, positive values for $n_{B}$ are also plausible in other magnetogenesis processes, like the electroweak phase transition (Grasso \& Rubinstein 2001; Kandus et al. 2011; Durrer \& Neronov 2013) and Higgs field gradients (Vachaspati 1991), or in the case of subsquent evolution of the
PMFs, for example via the chiral anomaly (Boyarsky et al. 2012; Rogachevskii et al. 2017; Schober et al. 2018)

As expected from Eq. (9), on large scales (small $k$ ), for $n_{B}=-2.9$ the slope is nearly 1 and increases for increasing $n_{B}$. Small-scale structures are thus strongly magnetically amplified and reach an amplitude larger than the one induced by inflation alone. On very small scales, the power spectrum is sharply quenched owing to the magnetic pressure and radiation viscosity damping. The total power spectrum is thus characterized by a bump at the smallest amplified scales, $k \sim 10-1000 h \mathrm{Mpc}^{-1}$, depending on the exact values of $n_{B}$ and $B_{\lambda}$. In Figs. 1 and 2 we link the wave number and the mass contained within a sphere of comoving Lagrangian radius $r_{l}$ at $z=0$ by defining the mass scale $M_{l}$ (the equivalent of the Jeans mass), assuming a background mean density given by the cosmological parameters (see e.g., Bullock \& Boylan-Kolchin 2017):

$$
M_{l}=\frac{4 \pi}{3} r_{l}^{3} \rho_{m}=\frac{\Omega_{m} H_{0}^{2}}{2 G} r_{l}^{3}=1.71 \times 10^{11} M_{\odot}\left(\frac{\Omega_{m}}{0.3}\right)\left(\frac{h}{0.67}\right)^{2}\left(\frac{r_{l}}{1 \mathrm{Mpc}}\right)^{3}
$$

The bumps precisely cover masses expected for the total mass of dwarf galaxies. Therefore, PMFs could significantly influence the formation and properties of those objects.

From Figs. 1 and 2 it appears that a steeper slope or a stronger amplitude would affect the power spectrum for $k<$ $10 \mathrm{hpc}^{-1}$. Such a modification is ruled out by the observations of the Lyman- $\alpha$ forest (Tegmark \& Zaldarriaga 2002; Pandey \& Sethi 2013).

\section{Simulations}

We performed a set of $\Lambda \mathrm{CDM}$ cosmological simulations with initial conditions that match the perturbed range of the total matter power spectrum described above. We used the same $\left(3.4 \mathrm{Mpc} h^{-1}\right)^{3}$ box adopted by Revaz \& Jablonka (2018) to study dwarf galaxies. The cosmology is the one described by the Planck Collaboration XIII (2016) with $\Omega_{\Lambda}=0.685, \Omega_{\mathrm{m}}=$ $0.315, \Omega b=0.0486, H_{0}=67.3 \mathrm{~km} \mathrm{~s}^{-1} \mathrm{Mpc}^{-1}, n_{s}=0.9603$, and $\sigma_{8}=0.829$. The relatively small box size used, prevents us from fully taking into account environmental effects such as those due to the proximity of a massive galaxy like the Milky Way.

Two types of simulations have been realized: (i) dark matter only (DMO) simulations of the entire box, used to compute the halo mass function and its dependency on the PMFs, and (ii) hydro-dynamical simulations of a selection of dwarf galaxies with the zoom-in technique. They allow us to check the effect of PMFs on the properties of stellar populations.

\subsection{Initial conditions}

The initial conditions were generated using the code MUSIC (Hahn \& Abel 2011). Instead of using, the Eisenstein \& Hu (1998), the default MUSIC power spectrum, we designed a special plug-in to use the total matter power spectrum obtained from the modified CAMB code. Compared to the Eisenstein \& Hu function, the plug-in includes baryonic pressure which leads to additional power on scales larger than $k \cong 100 \mathrm{~h} \mathrm{Mpc}^{-1}$, and results in an almost $10 \%$ difference in the mass content of structures formed in models without a magnetic field.

For the DMO simulations we used the resolution of level 9. This corresponds to $\left(2^{9}\right)^{3}$ particles, covering the entire box. For the hydro-dynamical simulations we used the zoom-in technique 
Table 1. Combination of explored parameters $B_{\lambda}$ and $n_{B}$ used for the seven simulations performed with a magnetically perturbed power spectrum.

\begin{tabular}{cccccccc}
\hline \hline Model & BQ.05n2.9 & BQ.05n2.7 & BQ.05n2.4 & BQ.05n2.1 & BQ.10n2.9 & BQ.20n2.9 & BQ.50n2.9 \\
\hline$B_{\lambda}[\mathrm{nG}]$ & 0.05 & 0.05 & 0.05 & 0.05 & 0.10 & 0.20 & 0.50 \\
$n_{B}$ & -2.9 & -2.7 & -2.4 & -2.1 & -2.9 & -2.9 & -2.9 \\
\hline
\end{tabular}

Notes. The models are referred to in the paper by the model names given below.

Table 2. Values of the fitting parameters used in Eq. (12) for different PMF models.

\begin{tabular}{cccccccc}
\hline \hline Model & BQ.05n2.9 & BQ.05n2.7 & BQ.05n2.4 & BQ.05n2.1 & BQ.10n2.9 & BQ.20n2.9 & BQ.50n2.9 \\
\hline $\mathrm{c}$ & 3.00 & 6.71 & 7.79 & 7.35 & 4.50 & 4.06 & 3.86 \\
$\mathrm{u}$ & 5.30 & 6.14 & 7.07 & 7.85 & 6.17 & 7.08 & 8.45 \\
$\mathrm{~s}$ & 0.80 & 0.60 & 0.57 & 0.52 & 0.70 & 0.70 & 0.67 \\
\hline
\end{tabular}

to gradually degrade the resolution from level 9 to level 6 outside the refined regions. With this setting the mass resolution of dark matter, gas, and stellar particles is $22^{\prime} 462 h^{-1} M_{\odot}, 4^{\prime} 096 h^{-1} M_{\odot}$, and $1^{\prime} 024 h^{-1} M_{\odot}$, respectively.

\subsection{Code evolution}

The simulations were run with the chemo-dynamical Tree/SPH code GEAR developed by Revaz \& Jablonka (2012, 2018), and Revaz et al. (2016). GEAR is a fully parallel code based on Gadget-2 (Springel 2005). It operates with individual and adaptive time steps (Durier \& Dalla Vecchia 2012) and includes recent SPH improvements such as the pressure-entropy formulation (Hopkins 2013). GEAR includes radiative gas cooling and redshift-dependent UV-background heating through the GRACKLE library (Smith et al. 2017). The metal line cooling is computed through the Cloudy code (Ferland et al. 2013) for solar abundances which is scaled according to the gas metallicity. Hydrogen self-shielding against the ionizing radiation is incorporated by suppressing the UV-background heating for gas densities above $n_{H}=0.007 \mathrm{~cm}^{-3}$ (Aubert \& Teyssier 2010). Star formation relies on the stochastic prescription proposed by Katz (1992), Katz et al. (1996). We used an efficiency of star formation parameter $c_{\star}=0.01$. The star formation recipe is supplemented by a modified version of the Jeans pressure floor (Hopkins et al. 2011; Revaz \& Jablonka 2018) by adding a nonthermal term in the equation of state of the gas to avoid any spurious gas fragmentation. The chemical evolution includes Type Ia and II supernovae ( $\mathrm{SNe}$ ) with yields taken from Tsujimoto et al. (1995) and Kobayashi et al. (2000), respectively. GEAR includes thermal blastwave-like feedback, for which $10 \%$ of the explosion energy of each SN, taken as $10^{51} \mathrm{erg}$, is released in the interstellar medium (i.e., the $\mathrm{SN}$ efficiency is $\epsilon=0.1$ ). The initial mass function (IMF) is sampled with the random discrete scheme (RIMFS) of Revaz et al. (2016). The released chemical elements are mixed using the smooth metallicity scheme (Okamoto et al. 2005; Tornatore et al. 2007; Wiersma et al. 2009).

\subsection{Set of simulations}

Each type of simulation, DMO, and zoom-in, is run eight times. A first run is performed without magnetically induced perturbations and seven others explore the effect of $B_{\lambda}$ and $n_{B}$ parameters with values given in Table 1 and corresponding to the total matter power spectra of Figs. 1 and 2.
Table 3. Dwarf galaxies simulated with the zoom-in technique.

\begin{tabular}{ccccl}
\hline \hline $\begin{array}{c}\text { Model } \\
\text { ID }\end{array}$ & $\begin{array}{c}L_{\mathrm{V}} \\
{\left[10^{6} L_{\odot}\right]}\end{array}$ & $\begin{array}{c}M_{\star} \\
{\left[10^{6} M_{\odot}\right]}\end{array}$ & $\begin{array}{c}M_{200} \\
{\left[10^{9} M_{\odot}\right]}\end{array}$ & SF Class \\
\hline h050 & 4.2 & 9.6 & 2.6 & Extended \\
h070 & 2.0 & 5.8 & 1.8 & Extended \\
h061 & 0.2 & 0.5 & 1.9 & Quenched \\
h141 & 0.2 & 0.6 & 0.8 & Quenched \\
h111 & 0.2 & 0.5 & 1.1 & Quenched \\
h122 & 0.1 & 0.4 & 1.0 & Quenched \\
h159 & 0.4 & 1.1 & 0.7 & Quenched \\
h168 & 0.1 & 0.3 & 0.6 & Quenched \\
h177 & 0.2 & 0.5 & 0.5 & Quenched \\
\hline
\end{tabular}

Notes. For each of them we provide the total $V$-band luminosity $L_{\mathrm{V}}$, total stellar mass $M_{\star}$, virial mass $M_{200}$, and the star formation type for the unperturbed models at $z=0$.

We selected nine halos from the 27 dwarfs presented in Revaz \& Jablonka (2018) and re-simulated them with the same zoom-in technique. The selection covers galaxies with $L_{\mathrm{V}}<$ $10^{7} L_{\odot}$, where $L_{\mathrm{V}}$ stands for the total $V$-band luminosity, and different star formation histories. Seven dwarfs are dominated by an old stellar population, being quenched by the UV background after at most four billion years, and two dwarfs are more massive, characterized by extended star formation histories. The list of the re-simulated dwarf galaxies is given in Table 3 with their basic properties as obtained in the unperturbed case at $z=0$ : total $V$-band luminosity $L_{\mathrm{V}}$, total stellar mass $M_{\star}$, and virial mass $M_{200}$, where the virial overdensity is 200 times the critical density.

All simulations are started at a redshift of 200, ensuring that the rms variance of the initial density field, $\sigma_{8}$, lies between 0.1 and 0.2 (Knebe et al. 2009). With the exception of the extreme cases, all halos reached $z=0$ (the reason for the crash will be discussed in the Appendix, Table A.1).

\section{Results}

\subsection{DMO simulations and halo mass function}

Figure 3 displays the dark matter surface density at $z=0$ of four models: the unperturbed $\Lambda \mathrm{CDM}$ model and three models with the magnetically induced bumps in the power spectrum that 


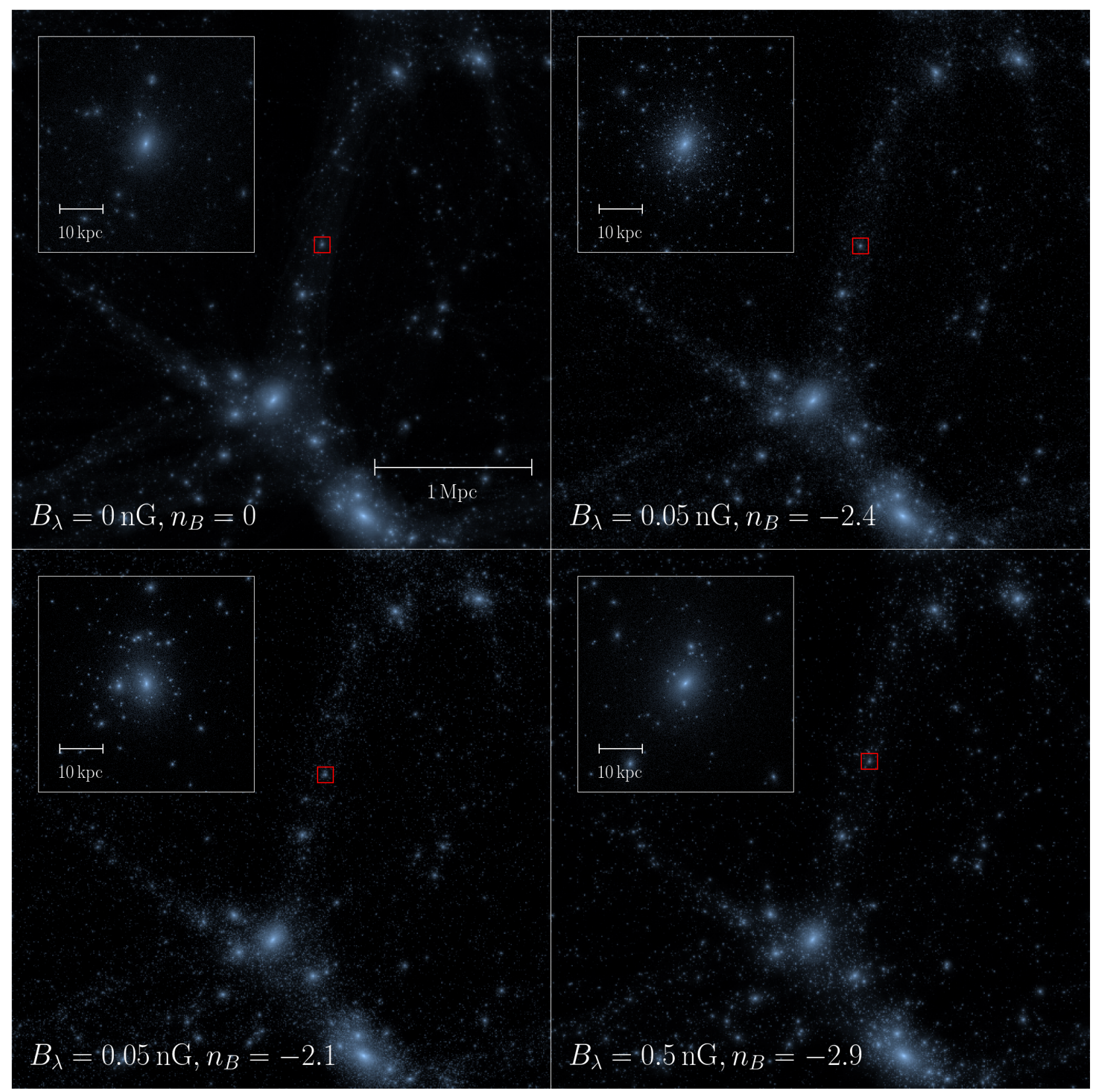

Fig. 3. Surface density projections of three DMO simulations at $z=0$ with different PMFs, as indicated at the bottom of each panel. Each plot shows the full box; the inset shows a magnification of the dwarf indicated by the red square.

peak at different mass scales: $\mathrm{B} 0.05 \mathrm{n} 2.4\left(M_{\text {peak }} \cong 5 \times 10^{6} M_{\odot}\right)$, BQ.05n2.1 $\left(M_{\text {peak }} \cong 3 \times 10^{7} M_{\odot}\right)$, and BQ.50n2.9 $\left(M_{\text {peak }} \cong\right.$ $\left.2 \times 10^{8} M_{\odot}\right)$. As expected, the small-scale bump increases the number of dark matter subhalos orbiting around dwarf galaxies. This number decreases with increasing mass of the halos. To quantify the effect of the bump observed in the total matter power spectrum, for all our DMO simulations we investigate the abundance of dark matter halos. We extracted dark matter halos using the Rockstar halo finder (Behroozi et al. 2013) which uses an adaptive hierarchical refinement of the friends-of-friends algorithm. All extracted halos consist of particle groups that are overdense with respect to the local background and contain a minimum of 100 bounded particles.

Figures 4 and 5 show the halo mass function: the number of dark matter halos, $\mathrm{d} N$, per unit mass interval, $\mathrm{d} M$. All mass functions are truncated below $3 \times 10^{5} M_{\odot}$, which corresponds to the limit of our mass resolution. While halos are detected with masses up to about $5 \times 10^{10} M_{\odot}$, the relatively small size of the box does not generate many of them. This makes the curve noisy, and we truncate it above $2 \times 10^{9} M_{\odot}$. Between these limits, the unperturbed power spectrum is nicely fitted by a powerlaw,

$$
\frac{\mathrm{d} N}{\mathrm{~d} M}=a_{0}\left(\frac{M}{m_{0}}\right)^{\alpha},
$$

with an amplitude of $a_{0}=8.79 \times 10^{7} / M_{\mathrm{vir}}=4.46 \times 10^{-5} M_{\odot}{ }^{-1}$, a pivot point of $m_{0}=10^{-5} M_{\mathrm{vir}}=1.97 \times 10^{7} M_{\odot}$ and a slope of $\alpha=-1.96$. These parameters perfectly match previous studies (see, e.g., Springel et al. 2008). The fit to the halo mass function is shown as a dashed gray line.

The bottom panels of Figs. 4 and 5 show the ratio between the magnetically modified halo mass function and the unperturbed one. Depending on the strength and the spectral index 


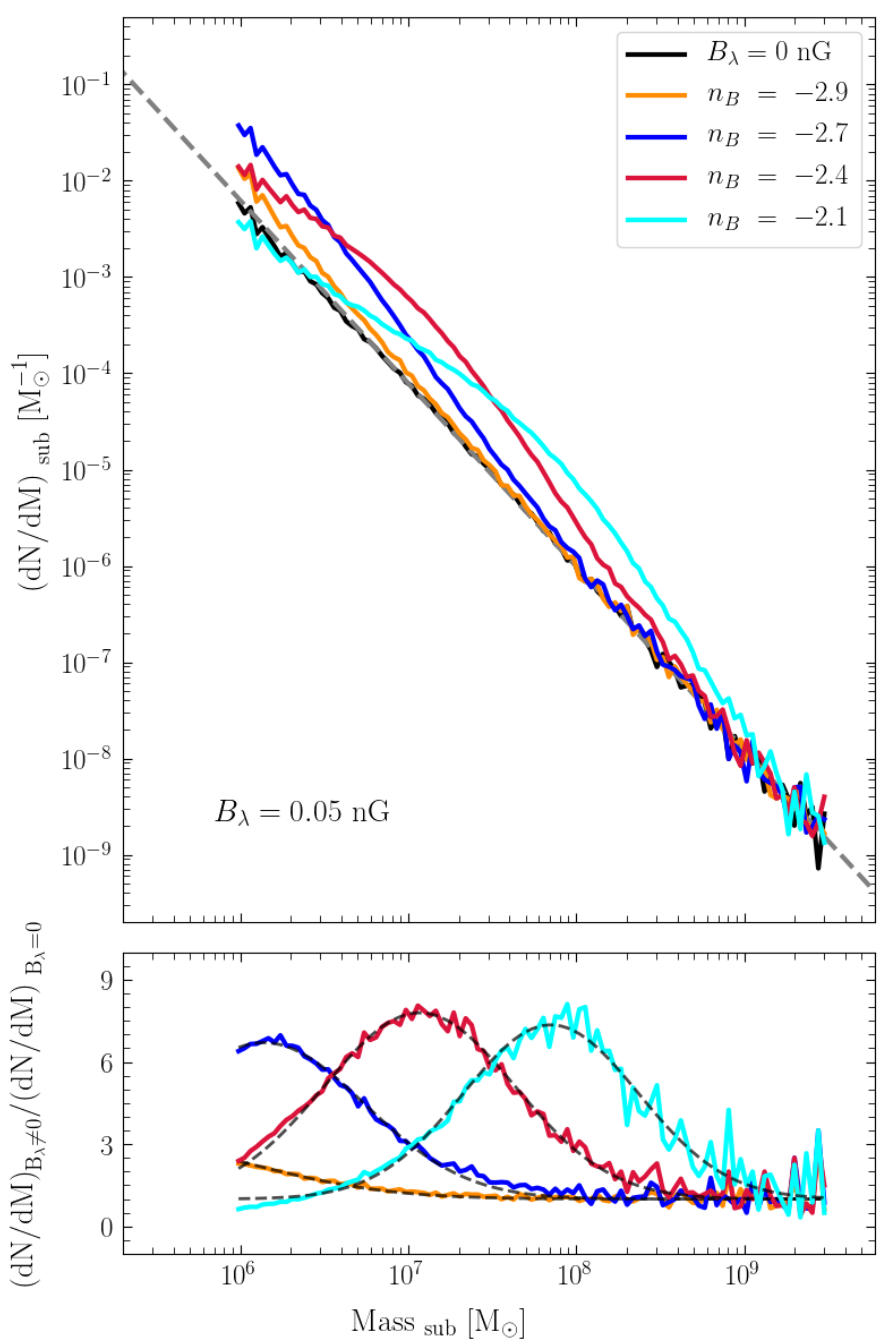

Fig. 4. Halo mass function. The halo mass is calculated within $R_{200}$ The count of halos is shown per logarithmic mass interval for different slope indexes $n_{B}$ with a constant magnetic field strength, $B_{\lambda}=0.05 \mathrm{nG}$. The dashed gray line shows a power law $\mathrm{d} N / \mathrm{d} M \sim M^{-1.96}$ fitted to the unperturbed power spectrum. The bottom panel shows the ratio of the halo mass function of each PMF model to the unperturbed model. The dashed lines correspond to the fit given by Eq. (12).

of the magnetic field, each PMF model affects the halo mass function in a different mass range below $\sim 10^{9} M_{\odot}$, reflecting the corresponding mass of the bump in the power spectrum. For a fixed magnetic amplitude, increasing the slope from $n_{B}=-2.7$ to -2.1 , shifts the bump from about $10^{6} M_{\odot}$ to $5 \times 10^{7} M_{\odot}$. On average, in a one dex mass range around the maximum of the bump, between 5 and 7 times more halos are present compared to the generic model without magnetic fields. Similarly, when the slope is fixed, increasing the magnetic amplitude, $B_{\lambda}$, shifts the bump upwards, increasing their number by a factor between 3 and 4.

We found the ratio of the perturbed to unperturbed halo mass functions to be well fit by a simple modified Gaussian function:

$$
\left(\frac{\mathrm{d} N}{\mathrm{~d} M}\right) /\left(\frac{\mathrm{d} N}{\mathrm{~d} M}\right)_{\text {unperturbed }}=(c-1) \exp \left[-\frac{\left(\log _{10}(M)-u\right)^{2}}{2 s^{2}}\right]+1
$$

(see values for $c, u$, and $s$ given in Table 2). The fit is shown by a dashed line in the bottom panel of Figs. 4 and 5. Equation (12)

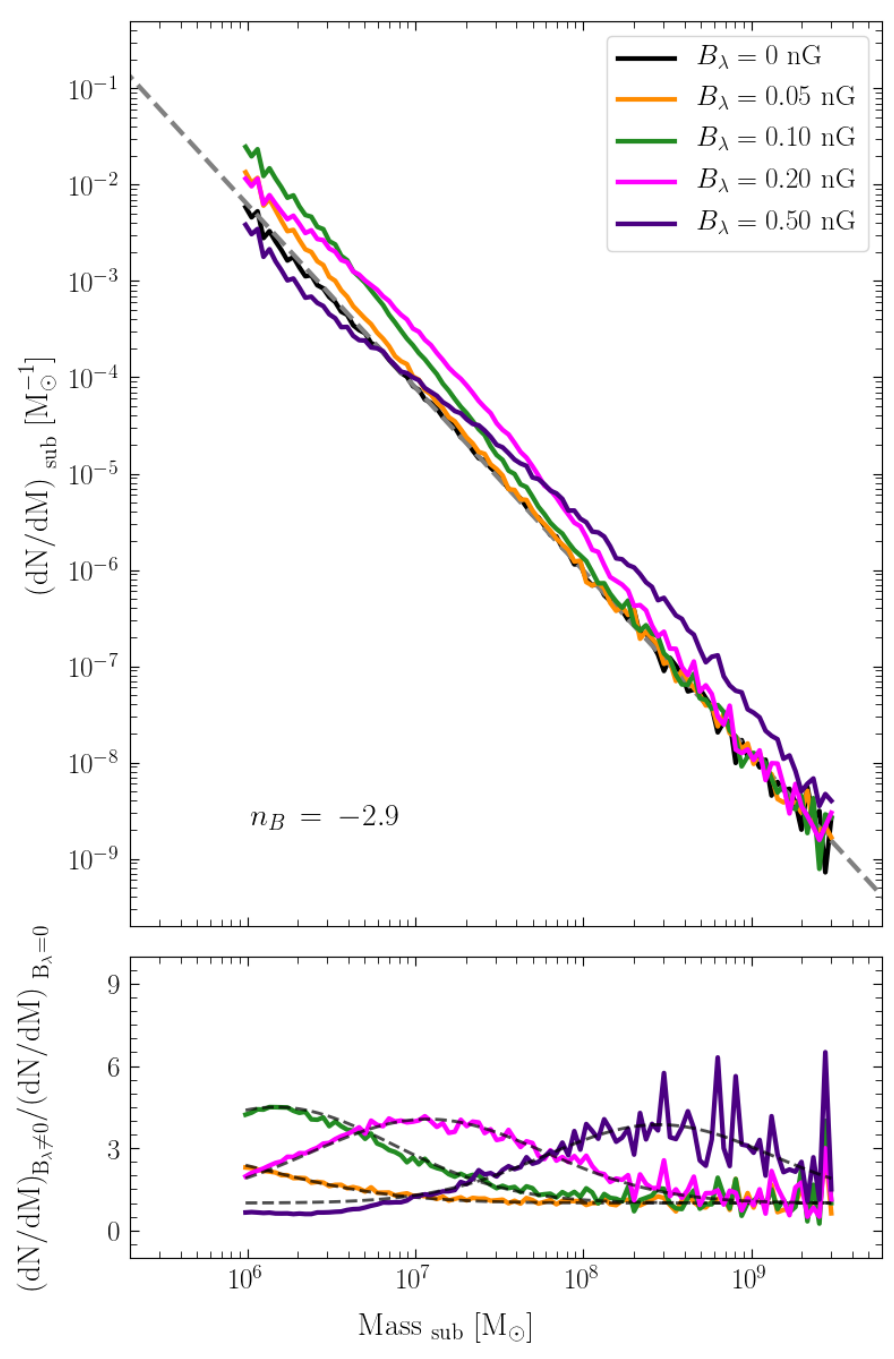

Fig. 5. Halo mass function. Same as Fig. 4, but for varying magnetic field strengths with the spectral slope kept constant to $n_{B}=-2.9$.

can be used to estimate analytically the magnetically perturbed halo mass function from a known unperturbed one. This is used in Sect. 4.3.2 to compare the predicted number of Milky Way satellites to the observed ones.

\subsection{Zoom-in simulations: physical properties}

From each zoom-in simulation, we first extracted the halo corresponding to the reference halo in the unperturbed model. Then, based on the positions and particle IDs, we extracted the analog of the same halo from the simulations in the perturbed models. For each extracted galaxy, we computed the following quantities defined inside one virial radius $R_{200}{ }^{1}$ : the $V$-band total stellar luminosity $L_{\mathrm{V}}$, the stellar mass $M_{\star}$, the virial mass $M_{200}$, the stellar LOS velocity dispersion $\sigma_{\text {LOS }}$, and the mode of the stellar metallicity distribution function $[\mathrm{Fe} / \mathrm{H}]$. The details of the procedures used to obtain these quantities are very similar to the ones used in Revaz \& Jablonka (2018). We reported these properties in Table A.1, Figs. A.1 and A.2.

1 While multiple dark subhalos are usually found within $R_{200}$ at $z=0$, the stellar component remains very compact $(<1 \mathrm{kpc})$. We checked that extracting quantities in a smaller region limited to the size of stellar component does not affect our results. 
Figure 6 displays the time evolution of halo h 070 for five different magnetically perturbed models (BQ .50n2.9, BQ .10n2.9, B0.05n2.1, BO.05n2.9), from $z=11.5$ down to $z=0$. They are compared to the unperturbed case. Table A.1 and Fig. 6 reveal the effect of PMFs, which can be seen as a sequence along the position and amplitude of the bump in the power spectrum.

In strongly perturbed models with high amplitude $B_{\lambda}$ or high spectral index $n_{B}$ (i.e., B0.50n2.9, B0.20n2.9, B0.05n2.1), the bump of the power spectrum peaks at mass ranges comparable to the mass scale of dwarf galaxies $\left(10^{7}\right.$ to $\left.10^{9} M_{\odot}\right)$. Those halos start clumping, accreting gas, and forming stars earlier. A deep potential well is quickly generated that prevents the gas reservoir from being evaporated due to the UV background. Contrary to the unperturbed case where the star formation history is quenched during the epoch of reionization, in the presence of PMFs the star formation is extended over longer periods, up to Gyrs in extreme cases (see Figs. 15 and 16), leading to more massive and brighter galaxies at $z=0$ with a younger stellar population. Not only the luminosity, but also the dynamics of the systems are affected, e.g., the central LOS velocity dispersion is much larger than its unperturbed analog, see Figs. 7 and 8. In extreme cases like BQ .50n2. 9 for more massive halos $\mathrm{h} 050$, h070, h061, h141 the star formation is so intense that it leads to a crash of the code. However, before the crash, as we will see, the models are already too bright and too metal rich to be compatible with observed dwarfs.

In weaker PMF models, namely B0.05n2.9, BO.05n2.7, as the magnetically induced bump of the power spectrum is in a mass range far below the mass scale of dwarf galaxies $\left(\lesssim 10^{6} M_{\odot}\right)$ and the amplitude of the bump is not significant, there are no noticeable changes in their built-up history and therefore in their properties. The small differences we see in Table A.1 and Figs. A.1 and A.2 are easily explained by stochasticity. For example, the star formation history is slightly expanded; indeed, the faint systems are very sensitive to perturbations.

With a bump at a mass of about $10^{7} M_{\odot}$, models BQ $.05 \mathrm{n} 2.4$ and $B Q .10 \mathrm{n} 2.9$ are intermediate cases. While their luminosity and metallicity are weakly affected, a noticeable difference appears in the number of subhalos orbiting each dwarf at $z=0$, as seen in Fig. 6. The increased number of dark matter halos could be an excellent marker of the existence of weak PMFs. Unfortunately, nowadays, they are out of reach to observations as their effect on the observed properties of dwarfs is negligible and they are not massive enough to be detected through gravitational lensing with current detection limits of about $10^{9} M_{\odot}$ (Vegetti et al. 2010). New forthcoming facilities like the Square Kilometer Array (SKA) will allow the detection of dark substructures with masses as low as $10^{6}-10^{7} M_{\odot}$ through their perturbation on gavitational arcs (McKean et al. 2015).

\subsubsection{Zoom-in simulations: scaling relations}

Figures 7 and 8 show the galaxy $V$-band luminosity $L_{\mathrm{V}}$ as a function of the LOS velocity dispersion $\sigma_{\text {LOS }}$, while Figs. 9 and 10 show their metallicity (traced by $[\mathrm{Fe} / \mathrm{H}]$ ) versus $L_{\mathrm{V}}$. As a comparison to the list of galaxies provided in the compilation of Local Group galaxies by McConnachie (2012), the sample we used is essentially restricted to the satellites brighter than $10^{5} \mathrm{~L}_{\odot}$. For the $[\mathrm{Fe} / \mathrm{H}]$ versus $L_{\mathrm{V}}$ relation, we used only galaxies that benefit from medium resolution spectroscopy with metallicity derived either from spectral synthesis or calcium triplet (CaT) calibration.

In Revaz \& Jablonka (2018) we showed that dwarfs emerging from a classical $\Lambda$ CDM Universe (i.e., unperturbed model in this work) nicely follow those relations over four orders of magnitude from $10^{5}$ to $10^{9} L_{\odot}$. The nine halos selected in this study from our sample of dwarf galaxies with the halo mass between $10^{8}$ and $10^{9}$ in the unperturbed model (black circles in Figs. 7-10) reproduce the chemo-dynamical properties of classical dwarf galaxies in this mass range observed around the Milky Way such as Ursa Minor, Draco, Sextans, or Sculptor. While the correlations between their $V$-band luminosity and line-of-sight velocity dispersion perfectly match those observed in the Local Group, we found our faintest dwarf to have a median metallicity slightly below the observed luminositymetallicity relation seen in Figs. 9 and 10. This discrepancy, which is not inherent to our models, might be due to the fact that both our IMF and SN yields are kept constant in this work.

The weakly or intermediate perturbed models (BQ.05n2.9, BQ.05n2 . 7, BQ.05n2.4, and BQ.10n2 .9) still stay on the scaling relations. It is worth noting that model BQ.05n2.7 suffers from a clear decrease in the luminosity (blue squares). In this model, due to the presence of the bump in the power spectrum at a mass of $\sim 10^{6} M_{\odot}$, many small subhalos are formed. Contrary to more perturbed models, they are not massive enough to form stars; however, this decrease in luminosity is not sufficient to rule out this model. The dwarfs emerging from intermediate case BQ.05n2. 4 with their velocity dispersion being increased, fall slightly off the relation. This increase is due to the presence of a larger number of small satellite halos that dynamically heat the stellar component.

The picture becomes very different, however, with stronger PMFs. All dwarfs simulated in models BO.05n2.1 and BO.50n2.9, exhibit star formation histories longer than $2 \mathrm{Gyr}$ (Figs. 15 and 16). They subsequently become brighter, but also denser, with strongly increased velocity dispersion. In the extreme case of model BQ.50n2.9, while galaxies are still following the $L_{\mathrm{V}}$ versus $\sigma_{\mathrm{LOS}}$ relation, they produce such a strong quantity of metals that they lie above the observed metallicityluminosity relation (Fig. 10).

\subsubsection{Luminosity versus halo mass}

As strongly magnetically perturbed models lead to an increase in the star formation rate, we expect the number of stars formed for a given halo mass to be modified. While not directly observed, the stellar mass to halo mass relation is important in particular to predict the number of visible satellites around the Milky Way (see Sect. 4.3.2).

To compute this relation, we extracted all halos found in the refined region of zoom-in simulations at $z=0$. We kept only halos containing at least ten stars and polluted by less than five percent of the boundary particles, i.e., particles coming from a region with a lower resolution. For each PMF model, we then plotted the stellar mass content of each halo as a function of its total mass, taken as $M_{200}$. The results are shown in Figs. 13 and 14 . We computed for every model an area delimited by the mean plus or minus one standard deviation of the distribution of galaxy stellar mass within each mass bins. In both figures, the gray area corresponds to the unperturbed model. For the unperturbed model, to increase the statistics, we used all dwarfs obtained from the sample of Revaz \& Jablonka (2018).

Models BQ.05n2.9, BO.10n2.9, BQ.05n2.7, and B0.05n2.4 display a stellar mass versus halo mass relation which lie within the bounded area of the unperturbed case. Model BQ.20n2.9 globally lies slightly above. However, the two strongly perturbed models, BO.50n2.9 and BO. $05 \mathrm{n} 2.1$ are 
M. Sanati et al.: Dwarf galaxies as a probe of Primordial Magnetic Fields

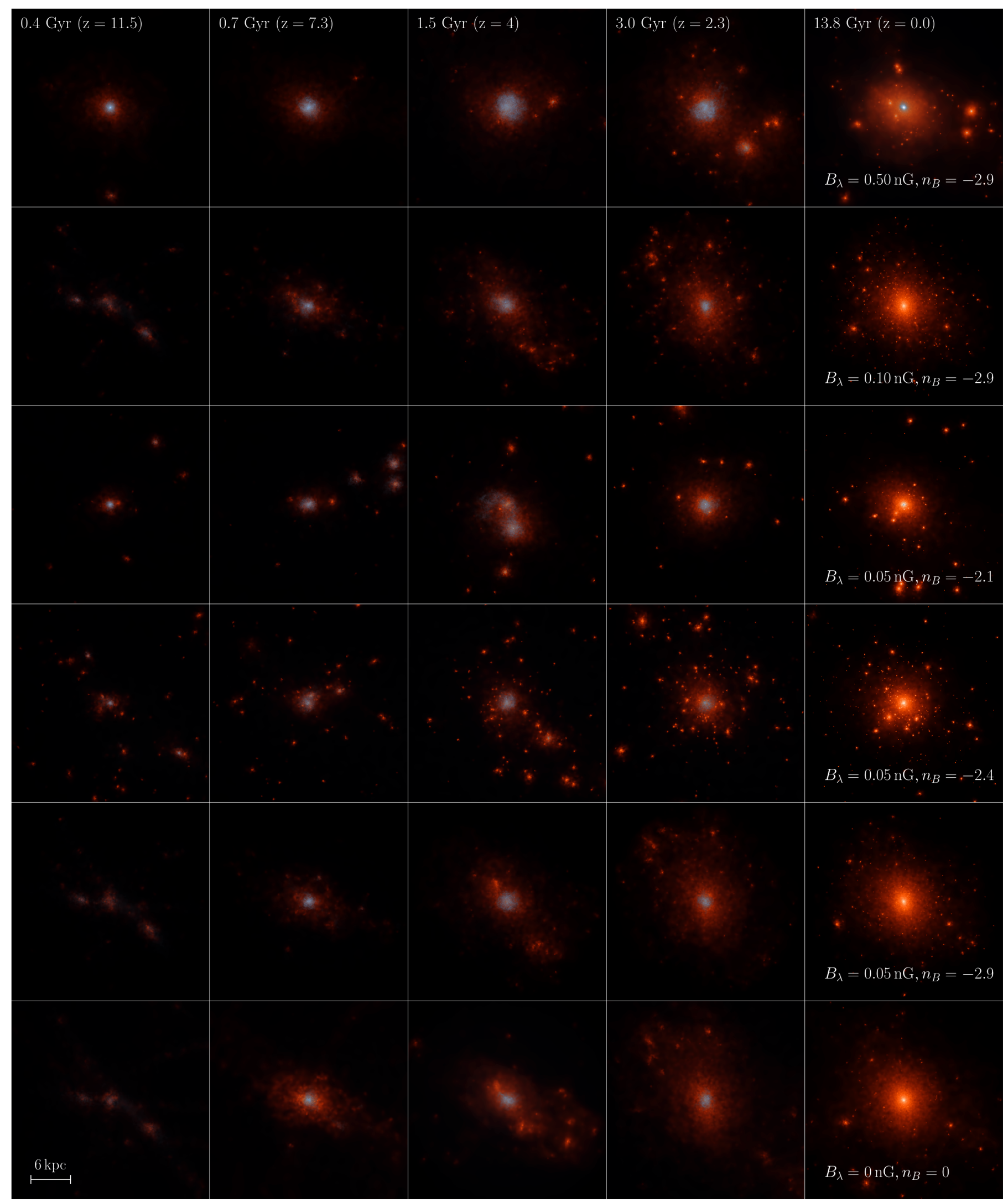

Fig. 6. Time evolution of halo h070 from $z \sim 11$ to $z=0$ for different magnetically perturbed models. The unperturbed model is shown at the bottom for comparison. In all images are shown the surface density (in comoving units) of the stars (in white), of the gas (in blue), and of the dark matter (in orange). Each box is 20 comoving kpc on a side. In the non-magnetic-field model at high redshifts the gas is dilute and is masked by the orange color of dark matter clumps, which start gathering and then at lower redshifts pull in neutral gas and form stars . However, in the extreme magnetic filed model the gas is more concentrated at high redshifts and a dwarf galaxy is formed very early on. 


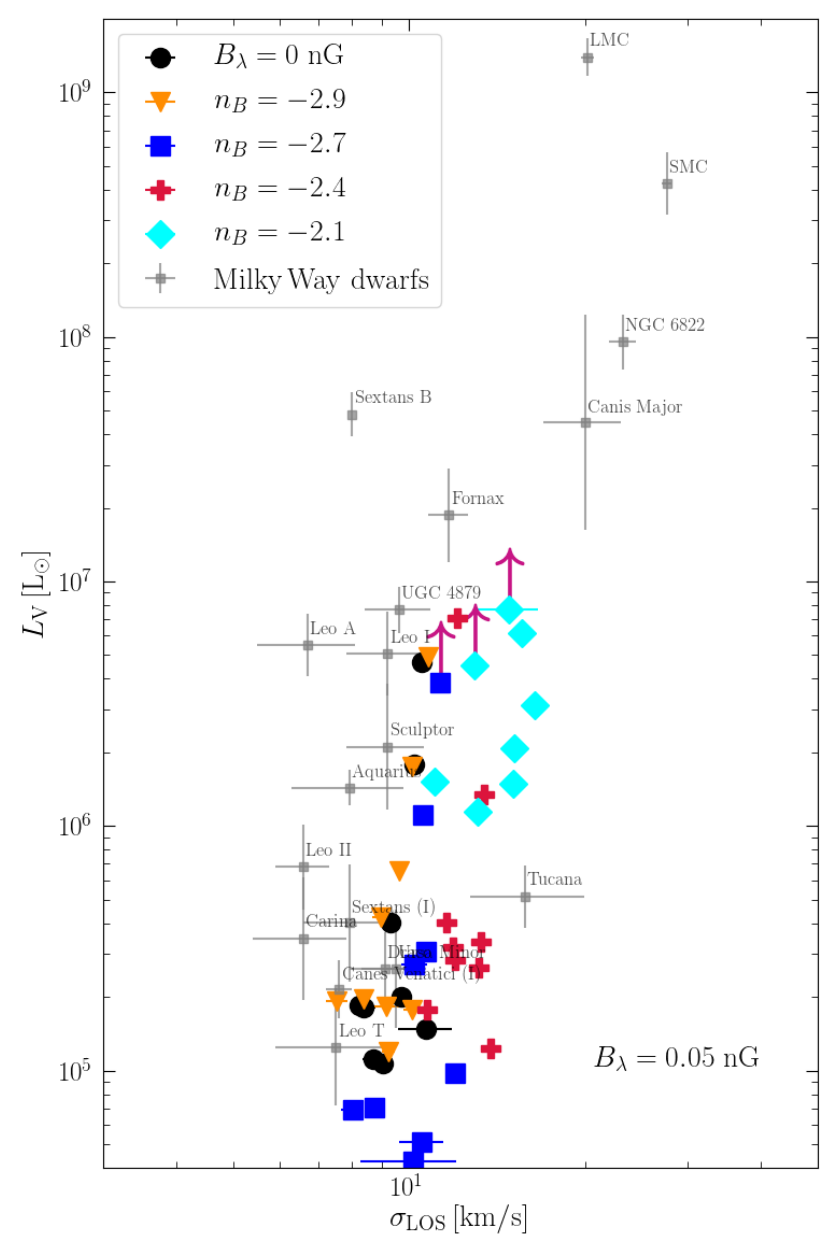

Fig. 7. $V$-band luminosity as a function of line-of-sight velocity dispersion in each model galaxy compared to the observational data of Milky Way satellites in black squares (see Sect. 4.2.1 for references). The purple arrows indicate models that did not reach $z=0$ and are expected to have a slightly brighter luminosity.

clearly above. Both produce a much larger quantity of stars, up to one dex, for a given halo mass.

\subsection{Cosmic star formation density and the reionization history of the Universe}

One striking impact of a strong PMF is to speed up the formation of dark matter halos on the dwarf galaxy scale, where the matter power spectrum is amplified (bumps in Figs. 1 and 2). This induces an earlier onset of star formation. This effect is qualitatively seen in Fig. 6 where at $z=11.5$ a dwarf is clearly formed in models BO.50n2.9, BO.05n2.1, and BO.05n2.4, while the gas has not yet finished collapsing in the other models.

To estimate this effect quantitatively, Figs. 11 and 12 show the cumulative mass of stars formed during the first Gyr. For each model the upper (resp. lower) edge of the colored area corresponds to the cumulative mass of the dwarf that forms the larger (resp. smaller) quantity of stars. For models BQ .50n2.9 and BO.20n2. 9 the onset of star formation starts before $z=50$, while for model B0.05n2.1 it starts before $z=25$, contrary to the unperturbed model in which all dwarfs form stars after $z=25$. In addition, the mean star formation rate is much stronger for models $B 0.50 \mathrm{n} 2.9$ and $B 0.05 \mathrm{n} 2.1$ than for the unperturbed model.

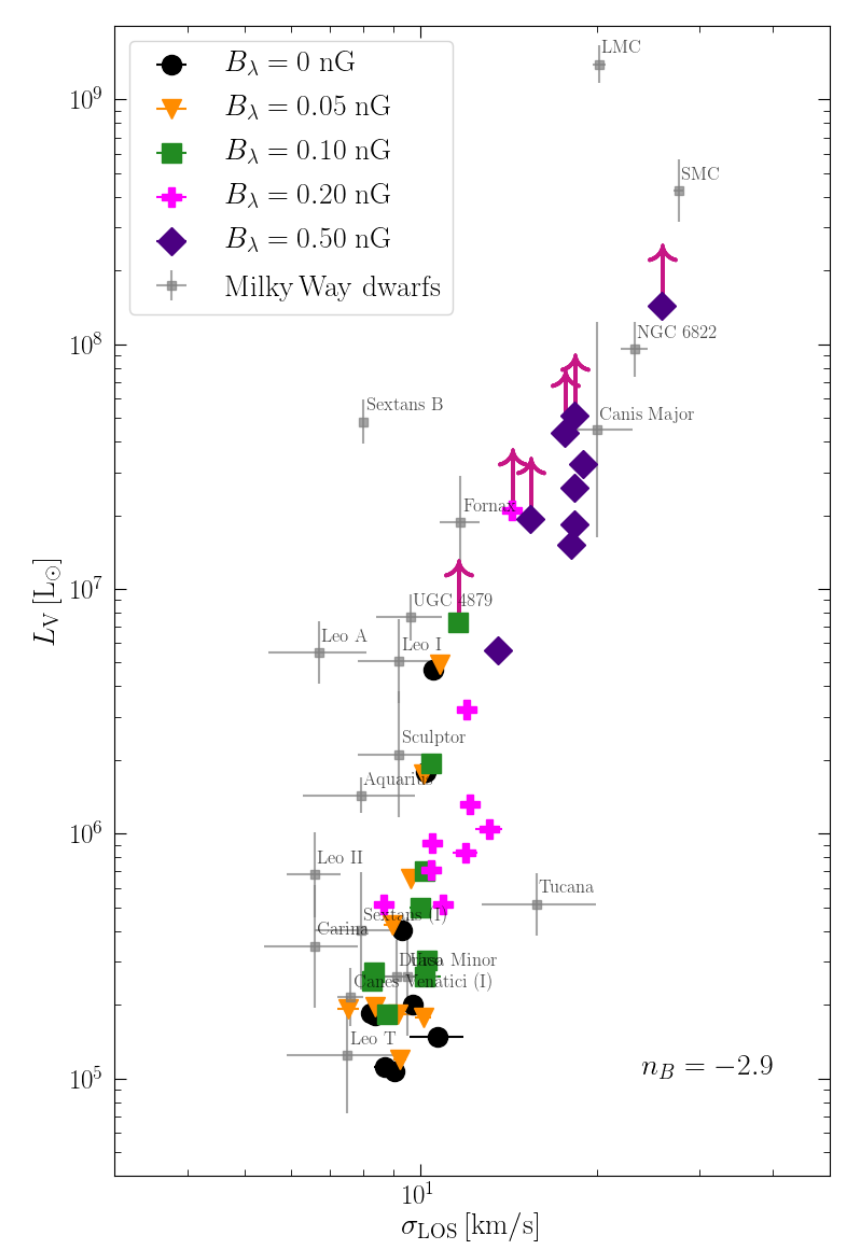

Fig. 8. $V$-band luminosity as a function of line-of-sight velocity dispersion. Same as Fig. 7, but for models with constant spectral slope and varying magnetic field strengths $B_{\lambda}$.

With such an early onset of star formation as well as enhancement of the star formation rate, these models will impact the reionization history of the Universe, i.e., the way the globally neutral inter galactic medium (IGM) becomes slowly ionized after being impacted by energetic UV photons generated by young and massive stars. The transition from a neutral IGM to an ionized one is now well constrained by different observations (see Fan et al. 2006a, for a review): by the Ly- $\alpha$ absorption by the IGM (the Gunn-Peterson effect) of high redshift quasars (Fan et al. 2006b; Schroeder et al. 2013; Davies et al. 2018a,b; Greig et al. 2019; Bañados et al. 2018; Durovčíková et al. 2020), by the gamma-ray bursts (Totani et al. 2006), by the prevalence, spectral properties or clustering of Ly- $\alpha$ emitting galaxies (Ouchi et al. 2010; Ono et al. 2012; Schenker et al. 2014; Tilvi et al. 2014), and by the cosmic microwave background (CMB) polarization through Thomson scattering (Spergel et al. 2003; Planck Collaboration Int. XLVII. 2016). In the following, we describe the method we used to estimate the evolution of the hydrogen ionized fraction from our simulations.

\subsubsection{Evolution of the hydrogen ionized fraction}

The time evolution of the ionized fraction of hydrogen $Q_{\mathrm{HII}}$ can be estimated using a simple differential equation (Madau et al. 1999; Springel \& Hernquist 2003; Kuhlen \& Faucher-Giguère 2012; Stoychev et al. 2019): 


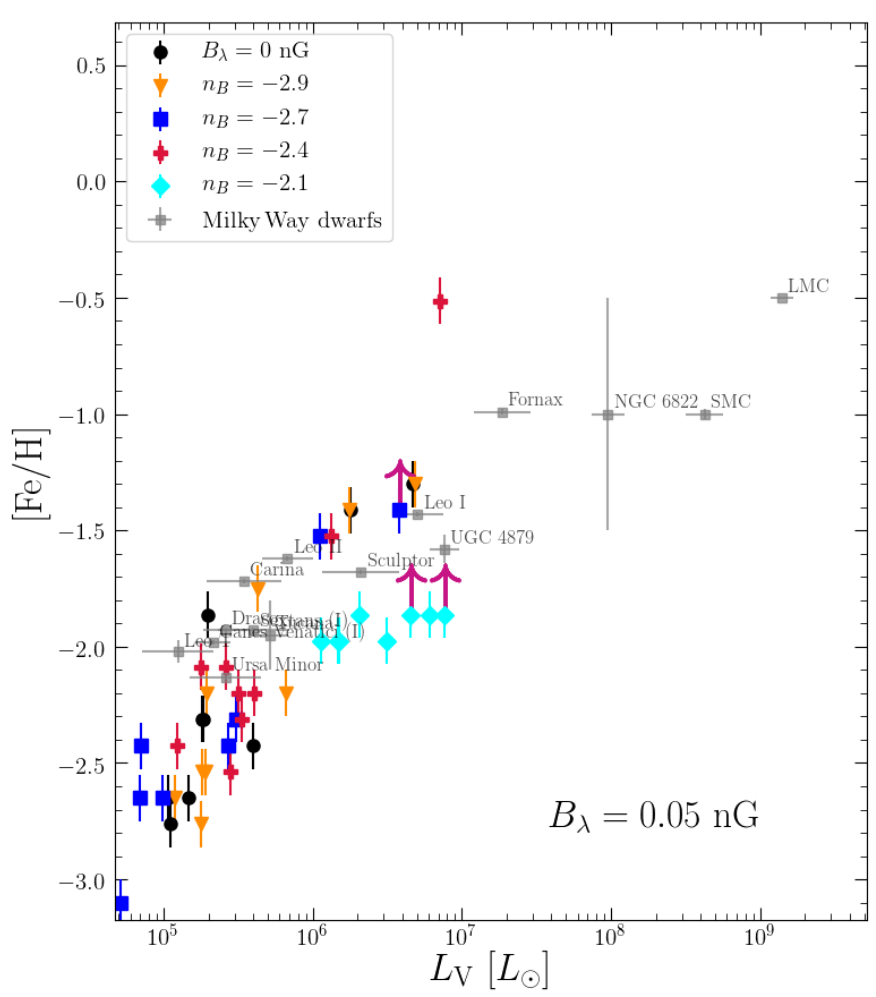

Fig. 9. Mean metallicity vs. stellar $V$-band luminosity. Each object corresponds to one model galaxy for which $[\mathrm{Fe} / \mathrm{H}]$ is computed as the median of the galaxy stellar metallicity distribution function. Milky Way satellites are identified with black squares (see Sect. 4.2.1 for references). The purple arrows indicate models that did not reach $z=0$ and are expected to have a slightly higher metallicity.

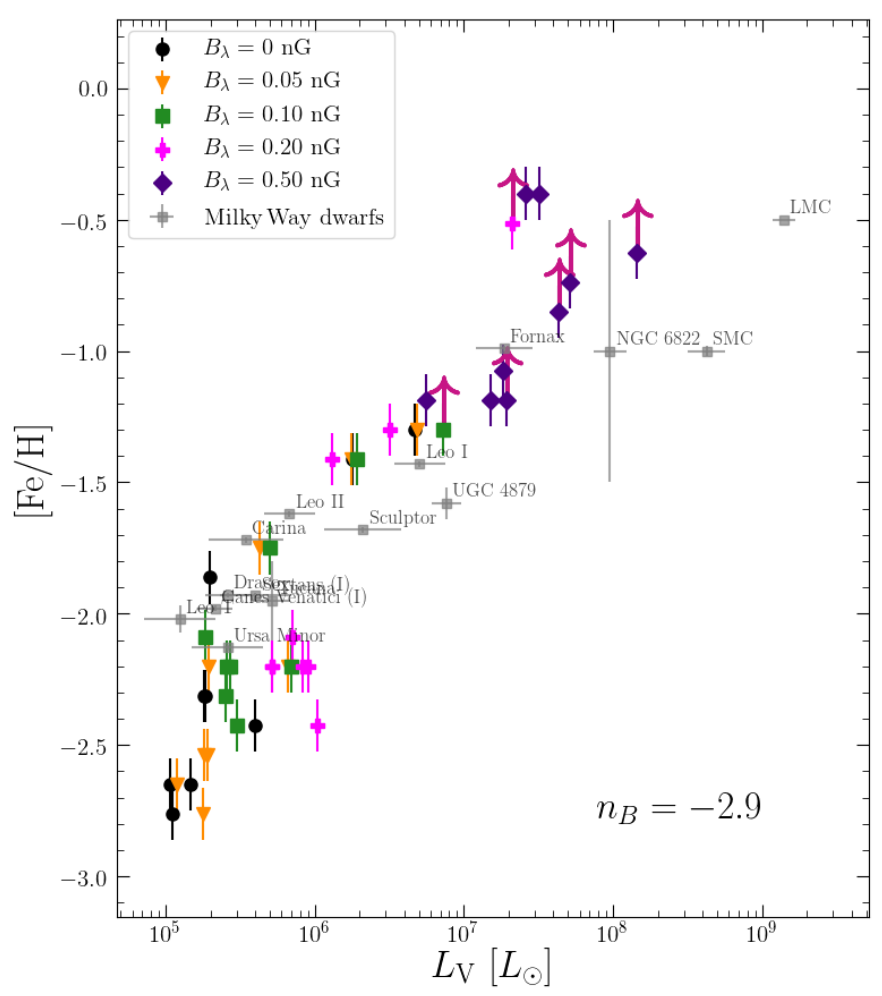

Fig. 10. Mean metallicity vs. stellar $V$-band luminosity. Same as Fig. 9 , but for models with constant $n_{B}$ and varying magnetic field strengths $B_{\lambda}$.

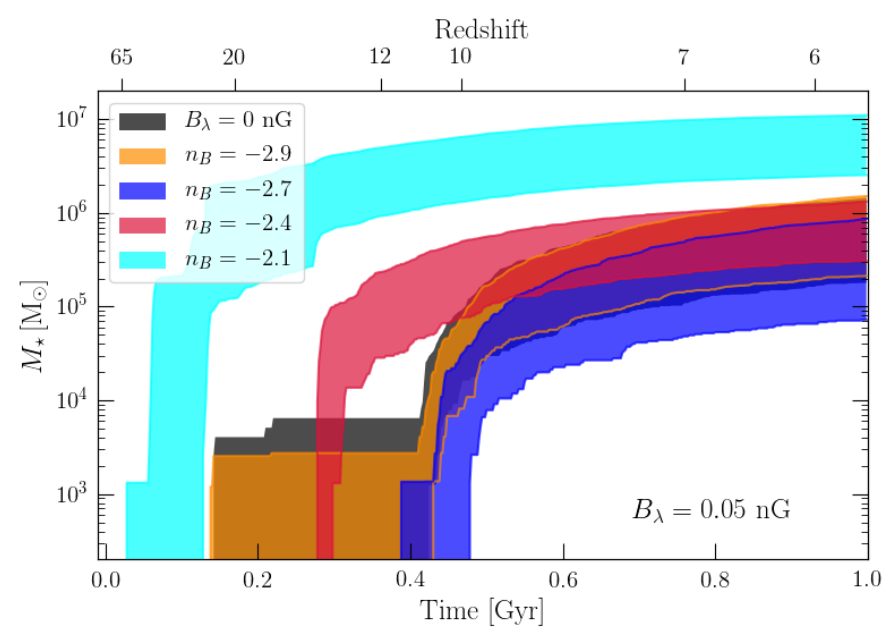

Fig. 11. Cumulative stellar mass in the first Gyr for different models with varying magnetic indices $n_{B}=-2.9$ to -2.1 . The amplitude of the magnetic field is kept constant at $B_{\lambda}=0.05 \mathrm{nG}$. The shaded area covers all nine halos simulated in various models.

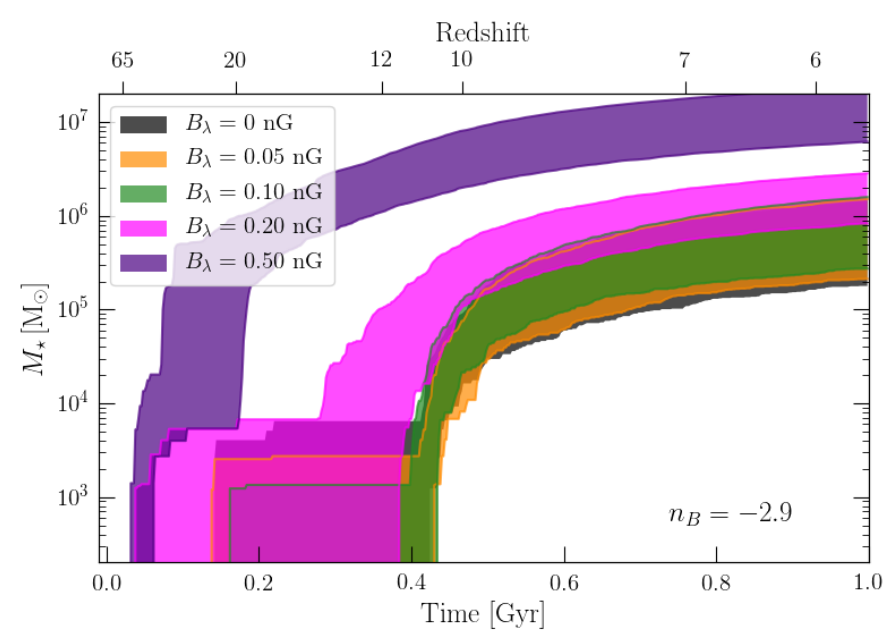

Fig. 12. Cumulative stellar mass in the first Gyr. Same as Fig. 11, but for a constant magnetic index $n_{B}=-2.9$ and a variety of magnetic amplitudes, from $B_{\lambda}=0.05$ to $B_{\lambda}=0.5 \mathrm{nG}$.

$\dot{Q}_{\mathrm{HII}}=\frac{\dot{n}_{\text {ion }}}{\bar{n}_{\mathrm{H}}}-\frac{Q_{\mathrm{HII}}}{t_{\text {rec }}}$.

The first term on the right-hand side describes the ionization source through the number of ionizing photons, while the second term, the sink term, is due to radiative cooling, leading to hydrogen recombination. Furthermore, $\dot{n}_{\text {ion }}$ is the global production rate of ionizing photons per unit volume, produced by young and massive stars. It is directly proportional to the cosmic star formation density $\rho_{\mathrm{SFR}}$

$\dot{n}_{\text {ion }}=\rho_{\text {SFR }} f_{\text {esc }} \chi_{\text {ion }}$,

where $f_{\text {esc }}$ represents the fraction of ionizing photons that escape star forming regions and $\chi_{\text {ion }}$ the ionizing photon production efficiency for a typical stellar population per unit time and per unit star formation rate. Moreover, $\bar{n}_{\mathrm{H}}$ is the mean comoving hydrogen number density defined through the baryonic fraction $\Omega_{\mathrm{b}}$, critical density $\rho_{\mathrm{c}}$, hydrogen mass fraction $X$, and hydrogen atom mass $m_{\mathrm{H}}$ : 
$\bar{n}_{\mathrm{H}}=\Omega_{\mathrm{b}} X \rho_{\mathrm{c}} / m_{\mathrm{H}}$

The sink term is due to the radiative recombination of protons with electrons described by the rate of change of the ionized hydrogen proper density $n_{\mathrm{HII}}$

$\frac{\mathrm{d}}{\mathrm{d} t} n_{\mathrm{HII}}=-n_{\mathrm{e}} n_{\mathrm{p}} \alpha_{\mathrm{HII}}(T)$,

where $\alpha_{\mathrm{HII}}(T)$ is the temperature dependent recombination rate coefficient (see Ferland et al. 1992, for tabulated values). Defining $X$ and $Y$ to be the mass fraction of hydrogen and helium, respectively, and $\eta$ the helium ionization degree $(0,1$, or 2$)$, we can replace the proper number density of electron $n_{\mathrm{e}}$ by $n_{\mathrm{H}}\left(Q_{\mathrm{HII}}+\eta \frac{Y}{4 X}\right)$ as well as the proper number density of proton $n_{\mathrm{p}}$ by $n_{\mathrm{H}} Q_{\mathrm{HII}}$. The recombination time $t_{\text {rec }}$ of Eq. (13) is then

$t_{\mathrm{rec}}=\frac{1}{C_{\mathrm{HII}}\left(Q_{\mathrm{HII}}+\eta \frac{Y}{4 X}\right) \bar{n}_{\mathrm{H}}(1+z)^{3} \alpha_{\mathrm{HII}}(T)}$,

where in Eq. (17), we replaced the proper hydrogen density by its comoving expression. An additional clumping factor $C_{\mathrm{HII}}$ is added to account for dense regions, self-shielded against ionizing photons that do not contribute to the recombination rate.

For each of our PMF models, we estimated the cosmic star formation density using the following procedure. First, using the Rockstar halo finder, we extracted dark matter halos from our DMO simulations, at a redshift of $6.3^{2}$. This provided us with a complete dark halo sample covering $\left(3.4 \mathrm{Mpc} h^{-1}\right)^{3}$ at a redshift near the end of the epoch of reionization (EoR). Next, using our zoom-in simulations, we extracted at the same redshift, all progenitors of all halos found at $z=0$ in the refined region ${ }^{3}$. This provided us with a sample of halos populated by galaxies for which we accurately know the star formation history. In the last step, we attributed to each dark halo (from the dark matter complete sample), a corresponding galaxy, matching their virial mass. This provided us with an estimate of the star formation in the full box and subsequently with the cosmic star formation density. The main difficulty of the method is correctting for the incompleteness of the galaxy samples extracted from the zoomin simulations. Indeed, these samples lack the most luminous objects. This does not limit our analysis as more massive and luminous systems are rare and do not dominate the ionzing photons production, and including them would lead to a slightly earlier reionization, worsening the situation.

To circumvent this difficulty, we used a simulation run with the same code and parameters (including the full baryonic physics), which used the same initial perturbation field covering the same cosmological volume, but with a homogeneous resolution corresponding to that of the refined region in the zoom-in simulations. This simulation allowed us to derive a reliable star formation history up to a redshift of 6.3 , where it stopped due to computational expenses. We then calibrated the star formation density derived from our unperturbed zoom-in simulation samples to the full box with a homogeneous resolution. We found a correction factor of 3 to be sufficient to recover the complete star formation history up to $z=6.3$. We then applied this factor to the star formation density of all others models.

\footnotetext{
2 The choice of this redshift is dictated by a calibration simulation that will be introduced later on. It is sufficient to constrain our models.

3 Halos at $z=0$ are all halos found in the refined region containing at least ten stellar particles, and being polluted by less than five percent of boundary particles, i.e., particles coming from a region with a lower resolution.
}

The evolution of the neutral hydrogen fraction $1-Q_{\mathrm{HII}}$ is then obtained from Eq. (13). Setting $X=0.76, Y=0.24$, $\eta=1$ (Faucher-Giguère et al. 2008), $C_{\mathrm{HII}}=3$ (Kaurov \& Gnedin 2015), and $\chi_{\text {ion }}=10^{53}$ photons $\left(\mathrm{M}_{\odot} \mathrm{yr}^{-1}\right)^{-1} \mathrm{~s}^{-1}$ (Stoychev et al. 2019), and using a fiducial IGM temperature of $2 \times 10^{4} \mathrm{~K}$ (Hui \& Haiman 2003), the data are nicely fitted by our unperturbed full box model, if the escape fraction is set to $12 \%$. Keeping this same parameters for all models, the evolution of the neutral hydrogen fraction $1-Q_{\mathrm{HII}}$ is displayed in Figs. 17 and 18.

Due to strongly enhanced star formation rates, the subsequently large amount of ionizing photons produced by models BQ.05n2 . 1, BQ.05n2 . 4, BQ.20n2 . 9, and BQ .50n2 . 9 lead to a total reionization of the universe at $z>9$. This is in total disagreement with observational constraints suggesting a reionization starting around $z=10$ and ending at about $z=6$ (Bolton \& Haehnelt 2007; Loeb \& Haiman 1997; Fan et al. 2000; Hu et al. 1999). The other models are in much better agreement. It should be noted here that our goal was not to precisely reproduce the observations. This requires a much more precise approach, for example self-consistently computing the radiative transfer of photons through the ISM and IGM. Our goal was rather to demonstrate that models with important magnetic field perturbation are far off the constraints by a comfortable margin, as is the case here.

In our approach, the growth of structures as well as the star formation histories of dwarfs are self-consistently followed. Our results corroborate results from Pandey et al. (2015), who estimated the Universe reionization using a semi-analytical estimation of the dark matter collapsed fraction, directly sensitive to matter power spectrum. They found that models with $B_{\lambda}>0.358,0.120,0.059 \mathrm{nG}$ with respectively $n_{B}=$ $-2.95,-2.9,-2.85$ are ruled out from existing constraints. Our approach also rules out models with $B_{\lambda} \geq 0.05 \mathrm{nG}$ and $n_{B} \geq-2.4$.

\subsubsection{Number of satellites in the Local Group}

In Sect. 4.1 we demonstrated how PMFs may lead to the formation of a larger number of low mass dark matter halos. This increase may potentially impact the number of observed luminous satellites around the Milky Way. In this section, we estimate the number of expected observed dwarf galaxies brighter than a given luminosity inside $300 \mathrm{kpc}$ and compare it with observations. For this purpose, we combined the halo mass functions obtained in Sect. 4.1 with the luminosity-halo mass relations of the zoom-in hydro-dynamical simulations of Sect. 4.2.2.

Assuming the halo mass function in the unperturbed model follows a power law (Eq. (11)), we can obtain an analytical relation of the cumulative abundance of halos $N(>M)$ by multiplying Eq. (11) by the magnetically induced perturbation (Eq. (12)) and integrating over the halo mass:

$$
\begin{aligned}
N(>M)= & -\frac{a}{b+1} M_{h}^{b+1}+ \\
& C\left[1-\operatorname{erf}\left(\frac{\log _{10}(M)-(b+1) s^{2} \ln (10)+u}{\sqrt{2} s}\right)\right],
\end{aligned}
$$

Here, erf is the standard error function and the constant $C$ is given by:

$$
\begin{aligned}
C= & \frac{1}{\sqrt{2}} \sqrt{\pi} a(c-1) s \ln (10) . \\
& \exp \left(\frac{(b+1) \ln (10)\left((b+1) s^{2} \ln (10)+2 u\right)}{2}\right) .
\end{aligned}
$$




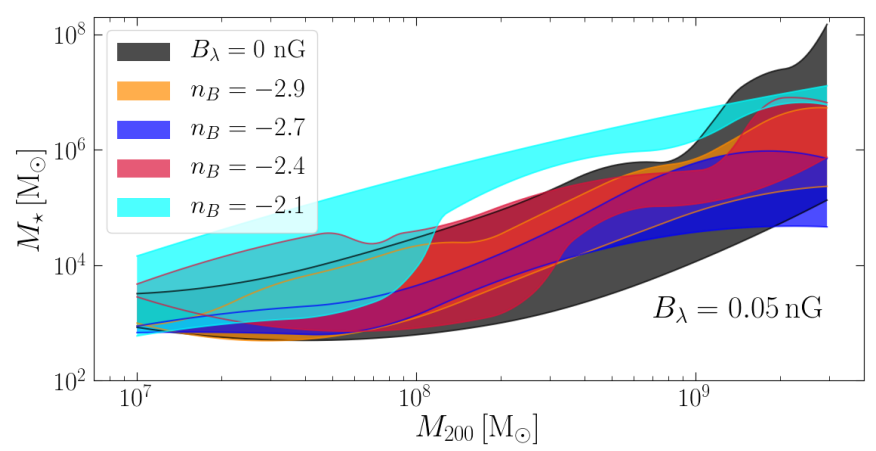

Fig. 13. Stellar mass vs. halo mass relation for different models with a variety of magnetic amplitude $B_{\lambda}$ and a constant slope index $n_{B}=-2.9$.

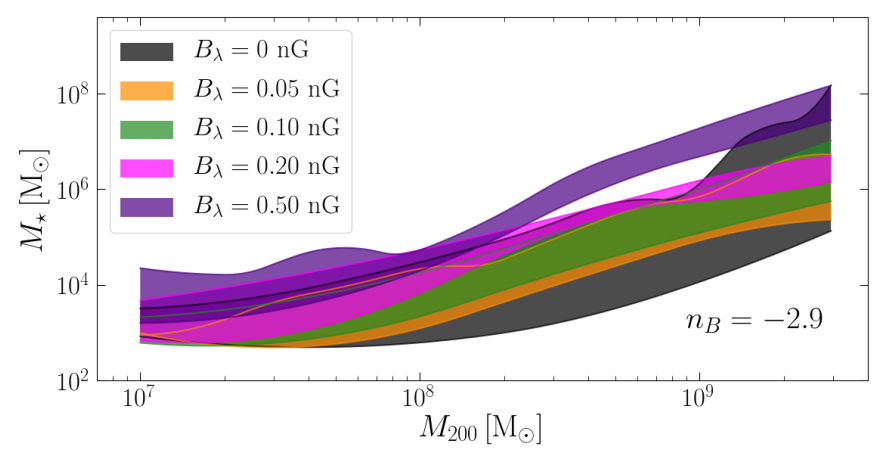

Fig. 14. Stellar mass vs. halo mass relation. Same as Fig. 13, but for models with constant $B_{\lambda}=0.05 \mathrm{nG}$ and different slope indexes $n_{B}$.

With this notation, the unperturbed case corresponds to $C=0$.

To reproduce a realistic halo mass function of the Local Group, which includes the perturbative effects of both Milky Way and Andromeda galaxy, we used the cumulative number of dark matter halos inside $300 \mathrm{kpc}$ predicted by the APOSTLE simulations (Sawala et al. 2017).

The corresponding mass function is obtained by a power law with a slope $b=-1.915$ taken by averaging the slopes of the four different radius bins given in Table 2 of Sawala et al. (2017). The amplitude $a=1.86 \times 10^{9}$ guarantees the existence of 800 dark matter halos with masses higher than $10^{7} M_{\odot}$ inside $300 \mathrm{kpc}$. This unperturbed cumulative halo distribution is then perturbed using Eq. (18). We note that for a perturbed halo distribution, the total number of halos with masses higher than $10^{7} M_{\odot}$ increases (up to about 4300 for model BQ.05n2.1), owing to the bump of the power spectrum that moves masses from smaller to larger scales. Inverting numerically Eq. (18), for each of our models, we randomly generated 1000 realizations of dark matter halos using a Monte Carlo approach. Relying on the halo mass versus luminosity relations (Figs. 13 and 14), we then assigned to each halo a stellar luminosity randomly chosen in the corresponding range showed by the shaded area. Having obtained a luminosity for each halo, we computed the cumulative number of satellites brighter than a given luminosity. Results are shown in Figs. 19 and 20.

Except for models B0.05n2.1, BQ.50n2.9, and B0.20n2.9, all models predict two times too many satellites for a luminosity higher than $10^{4} L_{\odot}$ where observed satellites show an intriguing dearth. On the contrary, models $\mathrm{BO} .05 \mathrm{n} 2.1, \mathrm{BO} .50 \mathrm{n} 2.9$, and BO.20n2.9 are clearly above the observations, predicting the existence of respectively 16 , 12 , and 4 times more satellites brighter than $10^{5} L_{\odot}$ compared to what is actually observed. This overabundance results from

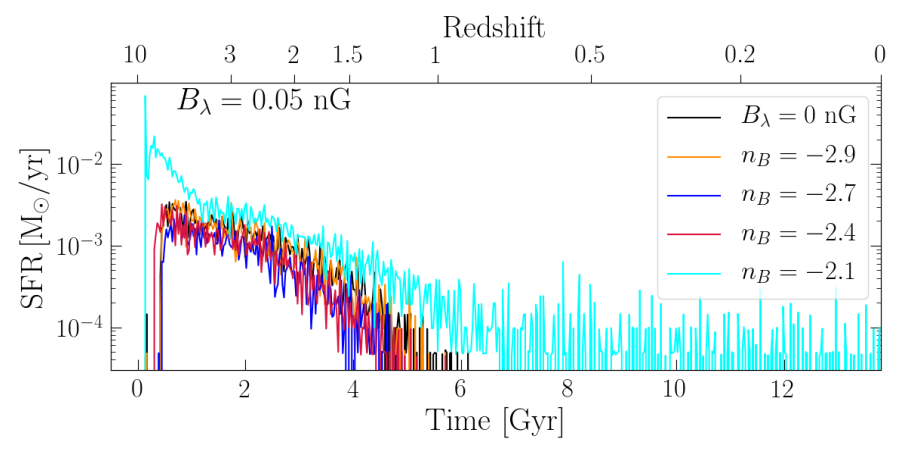

Fig. 15. Star formation rate of halo h070 for different models with varying magnetic indices $n_{B}=-2.9$ to -2.1 . The amplitude of the magnetic field is kept constant at $B_{\lambda}=0.05 \mathrm{nG}$.

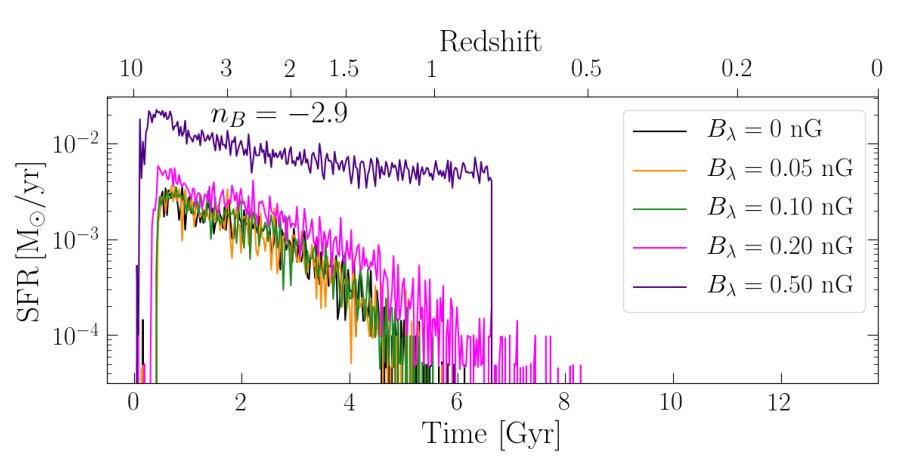

Fig. 16. Star formation rate. Same as Fig. 15, but for a constant magnetic index $n_{B}=-2.9$ and a variety of magnetic amplitudes, from $B_{\lambda}=0.05$ to $B_{\lambda}=0.5 \mathrm{nG}$.

the combined effect of a larger number of dark matter halos expected in the mass $10^{7}-10^{8} M_{\odot}$ (Figs. 4 and 5) and slightly brighter galaxies populating a given halo mass (Figs. 13 and 14).

We finally note that models with magnetic fields seems to underpredict the number of dwarfs brighter than $10^{6} L_{\odot}$. This is only the result of our incomplete sample which lacks bright dwarfs. The dark area corresponding to the unperturbed case has been obtained using the total sample of Revaz \& Jablonka (2018), which includes dwarfs up to $5 \times 10^{8} L_{\odot}$. In this case, an excellent match is obtained with observed dwarfs. The flattening of the curve below $10^{3} L_{\odot}$ is the result of the UV background heating that evaporates gas in the smallest halos and prevents star formation onset and is the result of our resolution limit.

\section{Conclusion}

We study the impact of primordial magnetic fields (PMFs) on the formation and evolution of dwarf galaxies through the modification of the $\Lambda \mathrm{CDM}$ matter power spectrum at the recombination era. Depending on the strength and the spectral index of the magnetic field, each PMF model affects the matter power spectrum in a different mass range. We examine a variety of PMF models by either changing the amplitude $\left(B_{\lambda}=0.05,0.10,0.20,0.50 \mathrm{nG}\right)$ or the slope $\left(n_{B}=-2.9,-2.7,-2.4,-2.1\right)$ of the magnetic power spectrum, keeping the other parameters constant.

We first run a set of DMO simulations covering a $\left(3.4 \mathrm{Mpc} h^{-1}\right)^{3}$ box with a resolution of $2 \times 512^{3}$ particles. Next, we re-simulate a set of nine halos extracted from the same volume, from redshift $z=200$ to $z=0$, using a zoom-in technique and including a full treatment of baryons with the stellar mass resolution of $1024 M_{\odot} h^{-1}$. Our sample of halos in the 


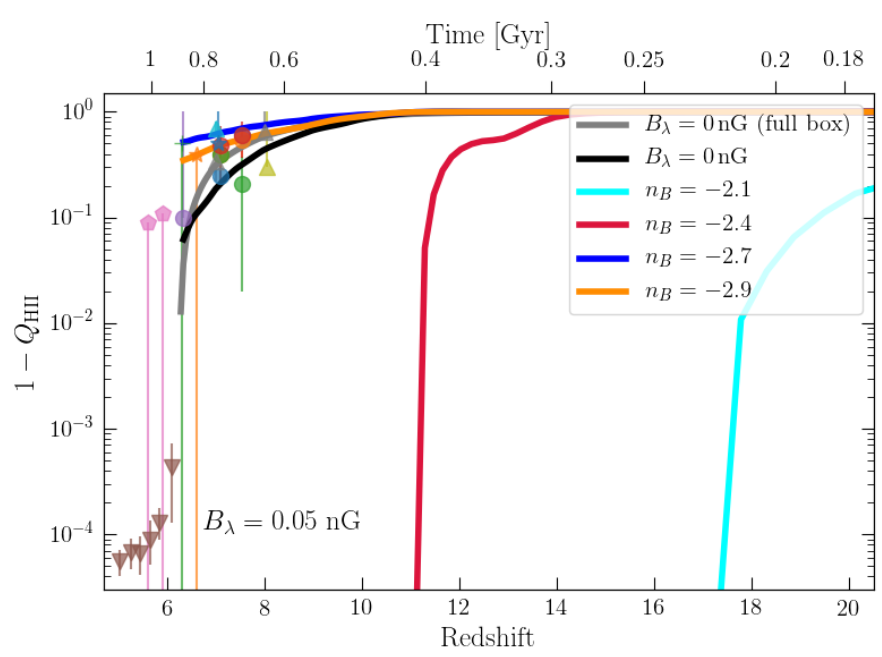

Fig. 17. Time evolution of the neutral hydrogen fraction $\left(1-Q_{\mathrm{HII}}\right)$ for models with different slope indexes $n_{B}$. The reference simulation (full box) is indicated by the gray curve. The model predictions are compared to observational constraints of the neutral hydrogen fraction from Ly- $\alpha$ absorption of quasars (Fan et al. 2006b) as brown downward triangles; (McGreer et al. 2015) pink pentagons; (Schroeder et al. 2013) purple, (Davies et al. 2018a,b) red, (Greig et al. 2017, 2019) green, (Bañados et al. 2018) orange, and (Durovčíková et al. 2020) blue circles; or gamma-ray bursts (Totani et al. 2006) as a green cross; and by Ly- $\alpha$ emitting galaxies (Ouchi et al. 2010) as an orange star, and (Ono et al. 2012) cyan, (Schenker et al. 2014) gray, and (Tilvi et al. 2014) yellow triangles.

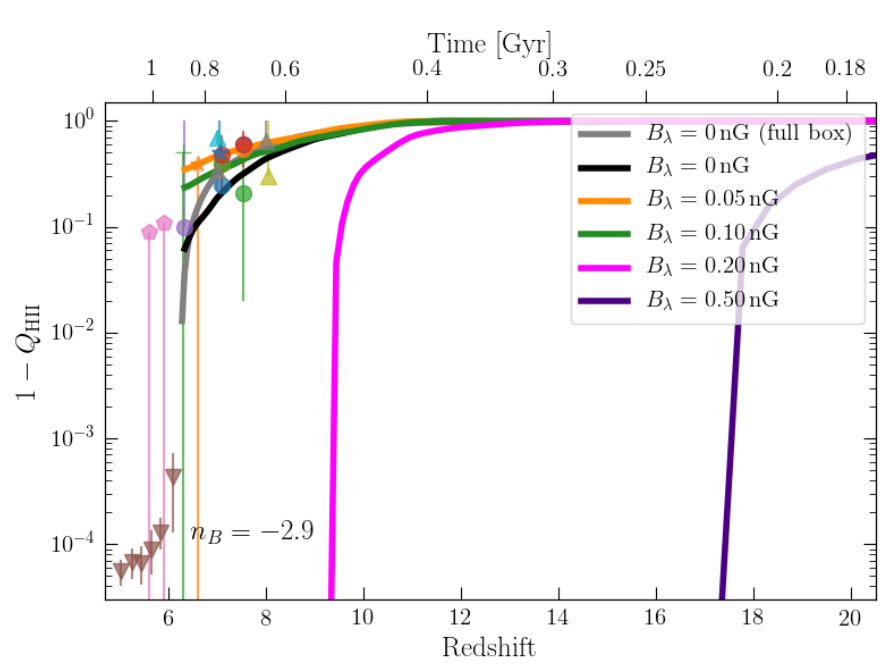

Fig. 18. Time evolution of the neutral hydrogen fraction. Same as Fig. 17, but for models with varying magnetic field strengths, from $B_{\lambda}=0.05$ to $0.50 \mathrm{nG}$.

unperturbed case give birth to dwarf spheroidals, seven of them having a quenched star formation history and two an extended history. Our results are summarized as follows:

1. To quantify the contribution of PMFs in the total matter power spectrum, we compute the halo mass function for all our DMO simulations. The ratio of perturbed to unperturbed mass function is well fitted by a simple Gaussian function. We show that increasing the magnetic amplitude $B_{\lambda}$, or the slope $n_{B}$, increases the number of halos around the maximum of the Gaussian function, up to a factor of 7 in the most extreme cases.

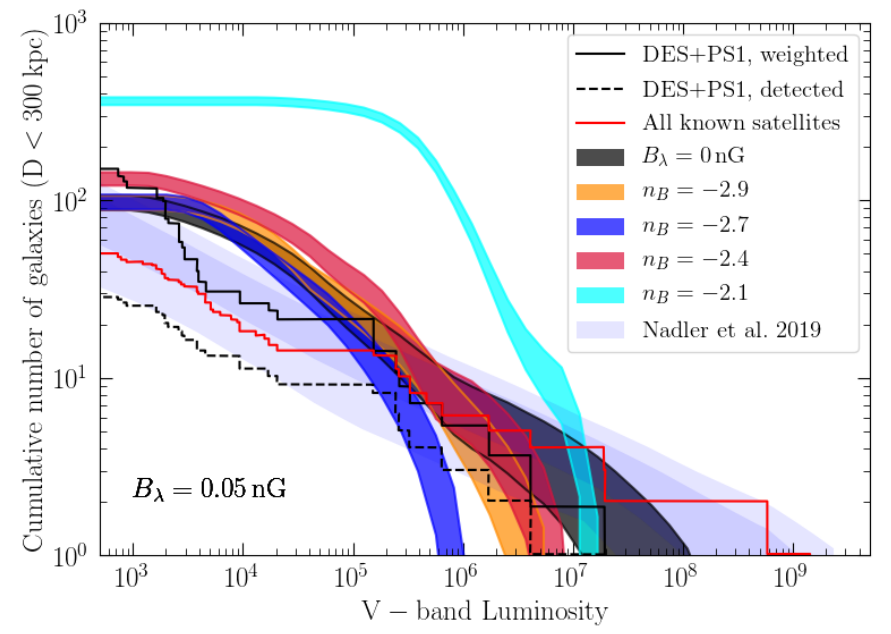

Fig. 19. Predicted cumulative number of satellites brighter than a given luminosity, within $300 \mathrm{kpc}$ around the Milky Way, for models with different slope indexes $n_{B}$. For each model the shaded area corresponds to the mean of thousand realizations of dark matter halos plus or minus one standard deviation. Our predictions are compared to the known satellites shown by the red curve. The dashed black line shows detected satellites in the Dark Energy Survey (DES) and Pan-STARRS1 (PS1) as presented in Drlica-Wagner et al. (2020). The solid black line shows the same data but volume corrected, assuming satellites are distributed isotropically. The light blue dashed regions show predictions from cosmological simulations combined with semi-analytical prescriptions (Nadler et al. 2019).

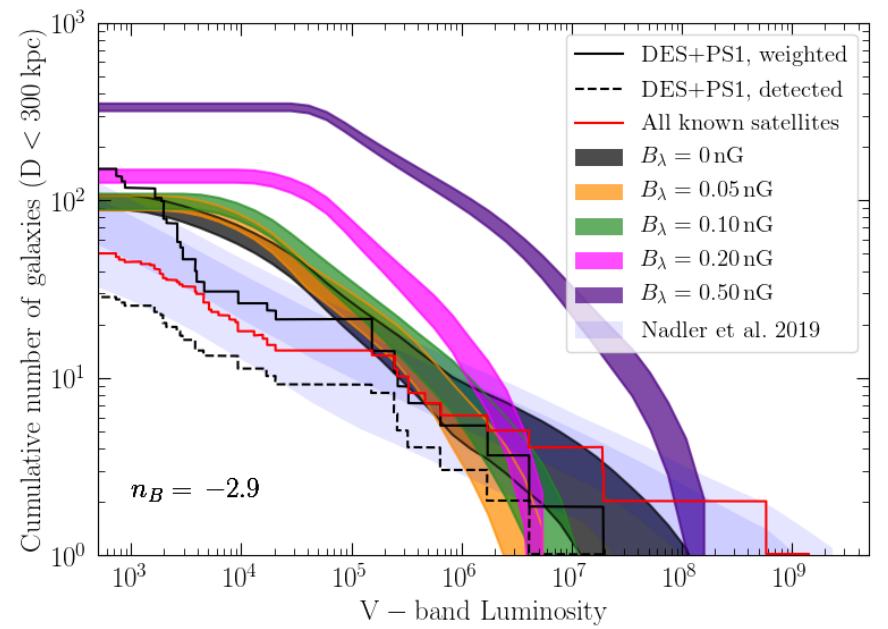

Fig. 20. Predicted cumulative number of satellites brighter than a given luminosity, within $300 \mathrm{kpc}$ around the Milky Way. Same as Fig. 19, but for models with varying magnetic field strengths from $B_{\lambda}=0.05$ to $0.50 \mathrm{nG}$.

2. We extract the observable properties of each galaxy at redshift $z=0$, including, the LOS velocity dispersion $\sigma_{\text {LOS }}$, the peak metallicity, and the total $V$-band luminosity $L_{\mathrm{V}}$ to compare them with well-observed scaling relations, such as the luminosity versus velocity dispersion and metallicity versus luminosity. Strongly perturbed models, with a high amplitude $\left(B_{\lambda}=0.5 \mathrm{nG}\right.$, $\left.n_{B}=-2.9\right)$ or a steep spectral index $\left(n_{B}=-2.1, B_{\lambda}=0.05 \mathrm{nG}\right)$ have more power in the mass range of dwarf galaxies, $10^{7}$ to $10^{9} M_{\odot}$. Consequently, in these models, galaxies form more stars and experience an extended star formation history, leading to brighter and more metal rich systems, incompatible with the 
observed Local Group scaling relations. On the contrary, the observed properties of the weakly perturbed models are not sensitively modified.

3. We show that strong magnetic models speed up the structure formation with an impact on the reionization of the Universe. We estimate the fraction of hydrogen ionized in the first Gyr of the Universe history and demonstrate that the earlier onset of star formation and the higher rate of star formation in these models induces a large amount of ionizing photons, enough to reionize the universe at redshifts higher than $z=9$, incompatible with the observational constraints for the EoR.

4. By combining the abundance of dark matter halos obtained from DMO simulations with the luminosity-halo mass function, we derive the number of luminous satellites expected around the Milky Way and show that with high magnetic amplitude or spectral index not only is the number of small dark matter halos increased in mass ranges $10^{7}$ and $10^{8} M_{\odot}$, but the stellar mass content for a given halo mass also increased, resulting in an overabundance of satellites brighter than $10^{5} L_{\odot}$, is contradiction with observations in the Local Group.

We conclude that galaxies simulated in weakly perturbed models resemble all physical properties of their counterparts in the unperturbed model. However, stronger models (e.g., $B_{\lambda}=$ $0.05 \mathrm{nG}$ with $n_{B}=-2.4$ or $n_{B}=-2.1$, and $B_{\lambda}=0.20 \mathrm{nG}$ or $B_{\lambda}=0.5 \mathrm{nG}$ with $\left.n_{B}=-2.9\right)$ may be ruled out due to the above-mentioned reasoning. Our results are consistent with cosmological observables, (e.g., CMB observations, weak gravitational lensing, Lyman $-\alpha$ data) that constrain the amplitude and the spectral index of the magnetic field power spectrum (Caprini et al. 2004; Lewis 2004; Kahniashvili et al. 2010; Pandey \& Sethi 2012; Trivedi et al. 2012; Shaw \& Lewis 2012; Pandey \& Sethi 2013; Kahniashvili et al. 2013b). We show that even smaller perturbed models can have a detectable impact on the structure formation. Further constraining PMFs could be considered by investigating the ultrafaint dwarf galaxies which are expected to be even more sensitive to perturbations with respect to their classical counterparts.

In this study we considered the contribution of PMFs in the $\Lambda \mathrm{CDM}$ matter power spectrum on mass scales comparable to dwarf galaxies. Regarding the possible direct impact of magnetic fields intrinsic to galaxies, on proto-stellar scales there are different procedures involved in boosting the star formation rate, namely by angular momentum loss due to the magnetic braking (Mouschovias \& Paleologou 1980; Ferreira et al. 2000; MartinAlvarez et al. 2020), or suppressing the star formation due to the magnetic pressure (Burkhart et al. 2009; Molina et al. 2012; Mocz et al. 2017; Sharda et al. 2020). Even so, the net effect is still an open question (see Krumholz \& Federrath (2019) for a recent review). It is certainly worth studying whether the modification to the star formation history due to the above procedures dominates over the impact of PMFs during the formation of the first structures. To answer this question it is necessary to include the full magneto-hydrodynamics treatment in our simulations. However, it is too computationally expensive at this moment. We leave this improvement for future studies.

Acknowledgements. We would like to thank Cheng Zhao, Loïc Hausammann, Mladen Ivkovic, and Florian Cabot for very useful discussions. We gratefully acknowledges financial support by Swiss government scholarship FCS. J.S acknowledges the funding from the European Unions Horizon 2020 research and innovation program under the Marie Skłodowska-Curie Grant No. 665667 and the support by the Swiss National Science Foundation under Grant No. 185863 K.E.K. acknowledges financial support by the Spanish Science Ministry gran PGC2018-094626-B-C22. We acknowledges the support by the International Space Science Institute (ISSI), Bern, Switzerland, for supporting and funding the international team "First stars in dwarf galaxies". This work was supported by the Swiss Federal Institute of Technology in Lausanne (EPFL) through the use of the facilities of its Scientific IT and Application Support Center (SCITAS). The simulations presented here were run on the Deneb clusters. The data reduction and galaxy maps have been performed using the parallelized Python pNbody package (http: //lastro.epfl. ch/projects/pNbody/).

\section{References}

Adshead, P., Giblin, J. T., Scully, T. R., \& Sfakianakis, E. I. 2016, J. Cosmol. Astropart. Phys., 2016, 039

Atek, H., Richard, J., Jauzac, M., et al. 2015, ApJ, 814, 69

Aubert, D., \& Teyssier, R. 2010, ApJ, 724, 244

Bañados, E., Venemans, B. P., Mazzucchelli, C., et al. 2018, Nature, 553, 473

Banerjee, R., \& Jedamzik, K. 2004, Phys. Rev. D, 70, 123003

Beck, R. 2001, Space Sci. Rev., 99, 243

Beck, R. 2015, A\&ARv., 24, 4

Beck, R., \& Wielebinski, R. 2013, in Magnetic Fields in Galaxies, eds. T. D. Oswalt, \& G. Gilmore, 5, 641

Behroozi, P. S., Wechsler, R. H., \& Wu, H.-Y. 2013, ApJ, 762, 109

Bernet, M. L., Miniati, F., Lilly, S. J., Kronberg, P. P., \& Dessauges-Zavadsky, M. 2008, Nature, 454, 302

Bertone, S., Vogt, C., \& Enßlin, T. 2006, MNRAS, 370, 319

Bolton, J. S., \& Haehnelt, M. G. 2007, MNRAS, 382, 325

Bouwens, R. J., Illingworth, G. D., Oesch, P. A., et al. 2015, ApJ, 811, 140

Boyarsky, A., Fröhlich, J., \& Ruchayskiy, O. 2012, Phys. Rev. Lett., 108, 031301

Boylan-Kolchin, M., Bullock, J. S., \& Kaplinghat, M. 2011, MNRAS, 415, L40

Boylan-Kolchin, M., Bullock, J. S., \& Kaplinghat, M. 2012, MNRAS, 422, 1203

Brandenburg, A., Kahniashvili, T., Mandal, S., et al. 2017a, Phys. Rev. D, 96, 123528

Brandenburg, A., Schober, J., Rogachevskii, I., et al. 2017b, ApJ, 845, L21

Bullock, J. S., \& Boylan-Kolchin, M. 2017, ARA\&A, 55, 343

Burkhart, B., Falceta-Gonçalves, D., Kowal, G., \& Lazarian, A. 2009, ApJ, 693, 250

Caprini, C., Durrer, R., \& Kahniashvili, T. 2004, Phys. Rev. D, 69, 063006

Chan, J. H. H., Schive, H.-Y., Woo, T.-P., \& Chiueh, T. 2018, MNRAS, 478, 2686

Chan, T. K., Kereš, D., Oñorbe, J., et al. 2015, MNRAS, 454, 2981

Choudhury, T. R., Ferrara, A., \& Gallerani, S. 2008, MNRAS, 385, L58

Clarke, T. E., Kronberg, P. P., \& Böhringer, H. 2001, ApJ, 547, L111

Davies, F. B., Hennawi, J. F., Bañados, E., et al. 2018a, ApJ, 864, 143

Davies, F. B., Hennawi, J. F., Bañados, E., et al. 2018b, ApJ, 864, 142

Dodelson, S. 2003, Modern cosmology (Elsevier)

Domcke, V., von Harling, B., Morgante, E., \& Mukaida, K. 2019, J. Cosmol. Astropart. Phys., 2019, 032

Drlica-Wagner, A., Bechtol, K., Mau, S., et al. 2020, ApJ, 893, 1

Durier, F., \& Dalla Vecchia, C. 2012, MNRAS, 419, 465

Durovčíková, D., Katz, H., Bosman, S. E. I., et al. 2020, MNRAS, 493, 4250

Durrer, R., \& Neronov, A. 2013, A\&ARv., 21, 62

Eisenstein, D. J., \& Hu, W. 1998, ApJ, 496, 605

Fan, X., White, R. L., Davis, M., et al. 2000, AJ, 120, 1167

Fan, X., Carilli, C. L., \& Keating, B. 2006a, ARA\&A, 44, 415

Fan, X., Strauss, M. A., Becker, R. H., et al. 2006b, AJ, 132, 117

Faucher-Giguère, C.-A., Lidz, A., Hernquist, L., \& Zaldarriaga, M. 2008, ApJ, 688,85

Ferland, G. J., Peterson, B. M., Horne, K., Welsh, W. F., \& Nahar, S. N. 1992, ApJ, 387, 95

Ferland, G. J., Porter, R. L., van Hoof, P. A. M., et al. 2013, Rev. Mex. Astron. Astrofis., 49, 137

Ferreira, J., Pelletier, G., \& Appl, S. 2000, MNRAS, 312, 387

Fitts, A., Boylan-Kolchin, M., Elbert, O. D., et al. 2017, MNRAS, 471, 3547

Fitts, A., Boylan-Kolchin, M., Bozek, B., et al. 2019, MNRAS, 490, 962

Fletcher, A., Beck, R., Shukurov, A., Berkhuijsen, E. M., \& Horellou, C. 2011,

MNRAS, 412, 2396

Flores, R. A., \& Primack, J. R. 1994, ApJ, 427, L1

Fujita, T., \& Durrer, R. 2019, J. Cosmol. Astropart. Phys., 2019, 008

Furlanetto, S. R., \& Loeb, A. 2001, ApJ, 556, 619

Giovannini, M., \& Shaposhnikov, M. 2000, Phys. Rev. D, 62, 103512

Gopal, R., \& Sethi, S. K. 2003, J. Astrophys. Astron., 24, 51

Governato, F., Weisz, D., Pontzen, A., et al. 2015, MNRAS, 448, 792

Govoni, F., \& Feretti, L. 2004, Int. J. Mod. Phys. D, 13, 1549

Grasso, D., \& Rubinstein, H. R. 2001, Phys. Rep., 348, 163

Greig, B., Mesinger, A., Haiman, Z., \& Simcoe, R. A. 2017, MNRAS, 466, 4239

Greig, B., Mesinger, A., \& Bañados, E. 2019, MNRAS, 484, 5094

Hahn, O., \& Abel, T. 2011, MNRAS, 415, 2101

Han, J. L. 2017, ARA\&A, 55, 111

Hanayama, H., Takahashi, K., Kotake, K., et al. 2005, ApJ, 633, 941 
Harvey, D., Revaz, Y., Robertson, A., \& Hausammann, L. 2018, MNRAS, 481, L89

Hausammann, L., Revaz, Y., \& Jablonka, P. 2019, A\&A, 624, A11

Hogan, C. J. 1983, Phys. Lett. B, 133, 172

Hopkins, P. F. 2013, Pressure-Entropy SPH: Pressure-Entropy Smooth-particle Hydrodynamics

Hopkins, P. F., Quataert, E., \& Murray, N. 2011, MNRAS, 417, 950

Hu, E. M., McMahon, R. G., \& Cowie, L. L. 1999, ApJ, 522, L9

Hui, L., \& Haiman, Z. 2003, ApJ, 596, 9

Ichiki, K., \& Takahashi, K. 2006, Astron. Herald, 99, 568

Jedamzik, K., \& Pogosian, L. 2020, ArXiv e-prints [arXiv:2004 . 09487]

Jedamzik, K., \& Saveliev, A. 2019, Phys. Rev. Lett., 123, 021301

Jedamzik, K., Katalinić, V., \& Olinto, A. V. 1998, Phys. Rev. D, 57, 3264

Jedamzik, K., Katalinic, V., \& Olinto, A. V. 2000, Phys. Rev. Lett., 85, 700

Kahniashvili, T., Tevzadze, A. G., Brand enburg, A., \& Neronov, A 2013a, Phys. Rev. D, 87, 083007

Kahniashvili, T., Maravin, Y., Natarajan, A., Battaglia, N., \& Tevzadze, A. G. 2013b, ApJ, 770, 47

Kahniashvili, T., Tevzadze, A. G., Sethi, S. K., Pandey, K., \& Ratra, B. 2010, Phys. Rev. D, 82, 083005

Kahniashvili, T., Brandenburg, A., Kosowsky, A., Mandal, S., \& Roper Pol, A 2020, IAU Gen. Assembly, 295

Kandus, A., Kunze, K. E., \& Tsagas, C. G. 2011, Phys. Rep., 505, 1

Katz, N. 1992, ApJ, 391, 502

Katz, N., Weinberg, D. H., \& Hernquist, L. 1996, ApJS, 105, 19

Kaurov, A. A., \& Gnedin, N. Y. 2015, ApJ, 810, 154

Kim, E.-J., Olinto, A. V., \& Rosner, R. 1996, ApJ, 468, 28

Klypin, A., Kravtsov, A. V., Valenzuela, O., \& Prada, F. 1999, ApJ, 522, 82

Knebe, A., Wagner, C., Knollmann, S., Diekershoff, T., \& Krause, F. 2009, ApJ, 698, 266

Kobayashi, C., Tsujimoto, T., \& Nomoto, K. 2000, ApJ, 539, 26

Kolb, E. W., \& Turner, M. S. 1990, S\&T, 80, 381

Krumholz, M. R., \& Federrath, C. 2019, Front. Astron. Space Sci., 6, 7

Kuhlen, M., \& Faucher-Giguère, C.-A. 2012, MNRAS, 423, 862

Kunze, K. E., \& Komatsu, E. 2014, J. Cosmol. Astropart. Phys., 01, 009

Lewis, A. 2004, Phys. Rev. D, 70, 043011

Loeb, A., \& Haiman, Z. 1997, ApJ, 490, 571

Lovell, M. R., Eke, V., Frenk, C. S., et al. 2012, MNRAS, 420, 2318

Madau, P., Haardt, F., \& Rees, M. J. 1999, ApJ, 514, 648

Mao, S. A., Carilli, C., Gaensler, B. M., et al. 2017, Nat. Astron., 1, 621

Martin, J., \& Yokoyama, J. 2008, J. Cosmol. Astropart. Phys., 2008, 025

Martin-Alvarez, S., Slyz, A., Devriendt, J., \& Gómez-Guijarro, C. 2020, MNRAS, 495, 4475

Mateo, M. L. 1998, ARA\&A, 36, 435

McBride, J., \& Heiles, C. 2013, ApJ, 763, 8

McConnachie, A. W. 2012, AJ, 144, 4

McGreer, I. D., Mesinger, A., \& D’Odorico, V. 2015, MNRAS, 447, 499

McKean, J., Jackson, N., Vegetti, S., et al. 2015, Advancing Astrophysics with the Square Kilometre Array (AASKA14), 84

Mocz, P., Burkhart, B., Hernquist, L., McKee, C. F., \& Springel, V. 2017, ApJ, 838,40

Molina, F. Z., Glover, S. C. O., Federrath, C., \& Klessen, R. S. 2012, MNRAS, 423, 2680

Moore, B. 1994, Nature, 370, 629

Moore, B., Ghigna, S., Governato, F., et al. 1999, ApJ, 524, L19

Mouschovias, T. C., \& Paleologou, E. V. 1980, ApJ, 237, 877

Nadler, E. O., Mao, Y.-Y., Green, G. M., \& Wechsler, R. H. 2019, ApJ, 873, 34

Nadler, E. O., Mao, Y.-Y., Wechsler, R. H., Garrison-Kimmel, S., \& Wetzel, A. 2018, ApJ, 859, 129

Naoz, S., \& Narayan, R. 2013, Phys. Rev. Lett., 111, 051303

Navarro, J. F., Frenk, C. S., \& White, S. D. M. 1996, ApJ, 462, 563

Navarro, J. F., Frenk, C. S., \& White, S. D. M. 1997, ApJ, 490, 493

Neronov, A., \& Vovk, I. 2010, Science, 328, 73

Newton, O., Cautun, M., Jenkins, A., Frenk, C. S., \& Helly, J. C. 2018, MNRAS, 479,2853

Oñorbe, J., Boylan-Kolchin, M., Bullock, J. S., et al. 2015, MNRAS, 454, 2092

Okamoto, T., Eke, V. R., Frenk, C. S., \& Jenkins, A. 2005, MNRAS, 363, 1299

Ono, Y., Ouchi, M., Mobasher, B., et al. 2012, ApJ, 744, 83

Ouchi, M., Shimasaku, K., Furusawa, H., et al. 2010, ApJ, 723, 869

Padmanabhan, T. 2002. Theoretical Astrophysics - Volume 3, Galaxies and Cosmology, 3

Pandey, K. L., \& Sethi, S. K. 2012, ApJ, 748, 27

Pandey, K. L., \& Sethi, S. K. 2013, ApJ, 762, 15

Pandey, K. L., Choudhury, T. R., Sethi, S. K., \& Ferrara, A. 2015, MNRAS, 451, 1692
Paoletti, D., Chluba, J., Finelli, F., \& Rubiño-Martín, J. A. 2019, MNRAS, 484, 185

Planck Collaboration XIII. 2016, A\&A, 594, A13

Planck Collaboration Int. XXVI. 2015, A\&A, 582, A29

Planck Collaboration Int. XLVII. 2016, A\&A, 596, A108

Pontzen, A., \& Governato, F. 2014, Nature, 506, 171

Ratra, B. 1992a, ApJ, 391, L1

Ratra, B. 1992b, ApJ, 391, L1

Read, J. I., Walker, M. G., \& Steger, P. 2018, MNRAS, 481, 860

Reiners, A. 2012, Liv. Rev. Sol. Phys., 9, 1

Revaz, Y., \& Jablonka, P. 2012, A\&A, 538, A82

Revaz, Y., \& Jablonka, P. 2018, A\&A, 616, A96

Revaz, Y., Arnaudon, A., Nichols, M., Bonvin, V., \& Jablonka, P. 2016, A\&A, 588, A21

Robertson, B. E., Ellis, R. S., Furlanetto, S. R., \& Dunlop, J. S. 2015, ApJ, 802, L19

Robishaw, T., Quataert, E., \& Heiles, C. 2008, ApJ, 680, 981

Rogachevskii, I., Ruchayskiy, O., Boyarsky, A., et al. 2017, ApJ, 846, 153

Ryu, D., Kang, H., Cho, J., \& Das, S. 2008, Science, 320, 909

Safarzadeh, M. 2018, MNRAS, 479, 315

Safarzadeh, M., \& Loeb, A. 2019, ApJ, 877, L27

Salvadori, S., Tolstoy, E., Ferrara, A., \& Zaroubi, S. 2014, MNRAS, 437, L26

Sawala, T., Frenk, C. S., Fattahi, A., et al. 2016, MNRAS, 457, 1931

Sawala, T., Pihajoki, P., Johansson, P. H., et al. 2017, MNRAS, 467, 4383

Schenker, M. A., Ellis, R. S., Konidaris, N. P., \& Stark, D. P. 2014, ApJ, 795, 20

Schober, J., Schleicher, D. R. G., \& Klessen, R. S. 2013, A\&A, 560, A87

Schober, J., Rogachevskii, I., Brandenburg, A., et al. 2018, ApJ, 858, 124

Schroeder, J., Mesinger, A., \& Haiman, Z. 2013, MNRAS, 428, 3058

Sethi, S. K., \& Subramanian, K. 2005, MNRAS, 356, 778

Sharda, P., Federrath, C., \& Krumholz, M. R. 2020, MNRAS, 497, 336

Shaw, J. R., \& Lewis, A. 2010, Phys. Rev. D, 81, 043517

Shaw, J. R., \& Lewis, A. 2012, Phys. Rev. D, 86, 043510

Silk, J. 1968, ApJ, 151, 459

Simon, J. D. 2019, ARA\&A, 57, 375

Smith, B. D., Bryan, G. L., Glover, S. C. O., et al. 2017, MNRAS, 466, 2217

Spergel, D. N., Verde, L., Peiris, H. V., et al. 2003, ApJS, 148, 175

Springel, V. 2005, MNRAS, 364, 1105

Springel, V., \& Hernquist, L. 2003, MNRAS, 339, 312

Springel, V., Wang, J., Vogelsberger, M., et al. 2008, MNRAS, 391, 1685

Stevenson, D. J. 2010, Space Sci. Rev., 152, 651

Stoychev, B. K., Dixon, K. L., Macciò, A. V., Blank, M., \& Dutton, A. A. 2019, MNRAS, 489, 487

Subramanian, K. 2016, Rep. Prog. Phys., 79, 076901

Subramanian, K., \& Barrow, J. D. 1998, Phys. Rev. Lett., 81, 3575

Tashiro, H., Silk, J., Langer, M., \& Sugiyama, N. 2009, MNRAS, 392, 1421

Tegmark, M., \& Zaldarriaga, M. 2002, Phys. Rev. D, 66, 103508

Tilvi, V., Papovich, C., Finkelstein, S. L., et al. 2014, ApJ, 794, 5

Tolstoy, E., Hill, V., \& Tosi, M. 2009, ARA\&A, 47, 371

Tornatore, L., Borgani, S., Dolag, K., \& Matteucci, F. 2007, MNRAS, 382, 1050

Totani, T., Kawai, N., Kosugi, G., et al. 2006, PASJ, 58, 485

Trivedi, P., Seshadri, T. R., \& Subramanian, K. 2012, Phys. Rev. Lett., 108, 231301

Tsujimoto, T., Nomoto, K., Yoshii, Y., et al. 1995, MNRAS, 277, 945

Turner, M. S., \& Widrow, L. M. 1988a, Phys. Rev. D, 37, 2743

Turner, M. S., \& Widrow, L. M. 1988b, Phys. Rev. D, 37, 2743

Vachaspati, T. 1991, Phys. Lett. B, 265, 258

Vegetti, S., Koopmans, L. V. E., Bolton, A., Treu, T., \& Gavazzi, R. 2010, MNRAS, 408, 1969

Verbeke, R., Papastergis, E., Ponomareva, A. A., Rathi, S., \& De Rijcke, S. 2017, A\&A, 607, A13

Vogelsberger, M., Zavala, J., Simpson, C., \& Jenkins, A. 2014, MNRAS, 444 3684

Vogt, C., \& Enßlin, T. A. 2005, A\&A, 434, 67

Wagstaff, J. M., \& Banerjee, R. 2015, Phys. Rev. D, 92, 123004

Wasserman, I. 1978a, ApJ, 224, 337

Wasserman, I. M. 1978b, Ph.D. Thesis, Harvard University

Wetzel, A. R., Hopkins, P. F., Kim, J.-H., et al. 2016, ApJ, 827, L23

Widrow, L. M. 2002, Rev. Mod. Phys., 74, 775

Wiersma, R. P. C., Schaye, J., Theuns, T., Dalla Vecchia, C., \& Tornatore, L. 2009, MNRAS, 399, 574

Wise, J. H., Demchenko, V. G., Halicek, M. T., et al. 2014, MNRAS, 442, 2560

Zucca, A., Li, Y., \& Pogosian, L. 2017, Phys. Rev. D, 95, 063506 


\section{Appendix A: Physical properties of model galaxies}

Table A.1. Physical properties of all model galaxies.

\begin{tabular}{|c|c|c|c|c|c|c|c|c|c|}
\hline $\begin{array}{l}\text { Model } \\
\text { ID }\end{array}$ & $\begin{array}{c}B_{\lambda} \\
{[\mathrm{nG}]}\end{array}$ & $\begin{array}{c}n_{B} \\
-\end{array}$ & $\begin{array}{c}L_{\mathrm{V}} \\
{\left[10^{6} L_{\odot}\right]}\end{array}$ & $\begin{array}{c}M_{\star} \\
{\left[10^{6} M_{\odot}\right]} \\
\end{array}$ & $\begin{array}{c}M_{200} \\
{\left[10^{9} M_{\odot}\right]}\end{array}$ & $\begin{array}{l}R_{200} \\
\mathrm{kpc}\end{array}$ & $\begin{array}{c}\sigma_{\mathrm{LOS}} \\
{\left[\mathrm{kms}^{-1}\right]}\end{array}$ & $\begin{array}{c}{[\mathrm{Fe} / \mathrm{H}]} \\
\operatorname{dex}\end{array}$ & $\begin{array}{r}\operatorname{Redshift}^{(a)} / \text { Time } \\
-/[\mathrm{Gyr}]\end{array}$ \\
\hline \multicolumn{10}{|c|}{$h 050$} \\
\hline BQ.00n0.0 & 0.00 & 0.0 & 4.66 & 10.01 & 2.66 & 33.14 & 10.52 & -1.30 & $0.00 / 13.8$ \\
\hline $\mathrm{B} 0.05 \mathrm{n} 2.9$ & 0.05 & -2.9 & 4.90 & 10.47 & 2.67 & 33.18 & 10.80 & -1.30 & $0.00 / 13.8$ \\
\hline BQ.05n2.7 & & -2.7 & 3.84 & 7.60 & 2.63 & 32.98 & 11.30 & -1.41 & $0.06 / 13.0$ \\
\hline BO. $05 \mathrm{n} 2.4$ & & -2.4 & 7.10 & 12.29 & 2.46 & 32.25 & 12.09 & -0.51 & $0.00 / 13.8$ \\
\hline $\mathrm{B} 0.05 \mathrm{n} 2.1$ & & -2.1 & -2.65 & 2.19 & - & - & - & - & $24.6 / 0.13$ \\
\hline $\mathrm{BQ} .10 \mathrm{n} 2.9$ & 0.10 & -2.9 & 7.33 & 13.76 & 2.62 & 32.94 & 11.61 & -1.30 & $0.04 / 13.3$ \\
\hline BQ.20n2.9 & 0.20 & & 20.99 & 31.93 & 2.51 & 32.49 & 14.35 & -0.51 & $0.11 / 12.3$ \\
\hline BQ.50n2.9 & 0.50 & & 143.40 & 147.52 & 3.51 & 36.33 & 25.83 & -0.62 & $0.62 / 7.8$ \\
\hline \multicolumn{10}{|c|}{ h070 } \\
\hline $\mathrm{B} 0.00 \mathrm{n} \theta .0$ & 0.00 & 0.0 & 1.78 & 5.13 & 1.80 & 29.08 & 10.20 & -1.41 & $0.00 / 13.8$ \\
\hline BQ.05n2.9 & 0.05 & -2.9 & 1.76 & 5.05 & 1.78 & 28.99 & 10.11 & -1.41 & $0.00 / 13.8$ \\
\hline $\mathrm{B} 0.05 \mathrm{n} 2.7$ & & -2.7 & 1.11 & 3.13 & 1.81 & 29.13 & 10.57 & -1.52 & $0.00 / 13.8$ \\
\hline $\mathrm{B} 0.05 \mathrm{n} 2.4$ & & -2.4 & 1.34 & 3.84 & 1.93 & 29.74 & 13.46 & -1.52 & $0.00 / 13.8$ \\
\hline $\mathrm{B} 0.05 \mathrm{n} 2.1$ & & -2.1 & 6.13 & 17.16 & 1.61 & 28.02 & 15.61 & -1.86 & $0.00 / 13.8$ \\
\hline $\mathrm{B} 0.10 \mathrm{n} 2.9$ & 0.10 & -2.9 & 1.93 & 5.52 & 1.79 & 29.00 & 10.44 & -1.41 & $0.00 / 13.8$ \\
\hline $\mathrm{BO} .20 \mathrm{n} 2.9$ & 0.20 & & 3.21 & 9.34 & 1.84 & 29.32 & 12.00 & -1.30 & $0.00 / 13.8$ \\
\hline BQ.50n2.9 & 0.50 & & 51.22 & 53.89 & 2.38 & 31.90 & 18.28 & -0.74 & $0.83 / 6.6$ \\
\hline \multicolumn{10}{|c|}{ h061 } \\
\hline $\mathrm{B} \theta .00 \mathrm{n} \theta .0$ & 0.00 & 0.0 & 0.20 & 0.50 & 1.95 & 29.85 & 9.72 & -1.86 & $0.00 / 13.8$ \\
\hline BQ.05n2.9 & 0.05 & -2.9 & 0.18 & 0.43 & 1.96 & 29.91 & 10.10 & -2.76 & $0.00 / 13.8$ \\
\hline $\mathrm{B} 0.05 \mathrm{n} 2.7$ & & -2.7 & 0.04 & 0.09 & 1.98 & 30.00 & 10.16 & -3.89 & $0.00 / 13.8$ \\
\hline BO. $05 \mathrm{n} 2.4$ & & -2.4 & 0.28 & 0.75 & 1.79 & 29.02 & 12.00 & -2.54 & $0.00 / 13.8$ \\
\hline BO. $05 \mathrm{n} 2.1$ & & -2.1 & 7.68 & 13.37 & 1.80 & 29.05 & 14.81 & -1.86 & $0.75 / 7.0$ \\
\hline BQ.10n2.9 & 0.10 & -2.9 & 0.30 & 0.74 & 1.94 & 29.82 & 10.25 & -2.42 & $0.00 / 13.8$ \\
\hline BO.20n2.9 & 0.20 & & 1.05 & 2.75 & 2.02 & 30.23 & 13.10 & -2.42 & $0.00 / 13.8$ \\
\hline BO. $50 \mathrm{n} 2.9$ & 0.50 & & 43.60 & 48.94 & 2.59 & 32.82 & 17.66 & -0.85 & $0.69 / 7.4$ \\
\hline \multicolumn{10}{|c|}{ h141 } \\
\hline $\mathrm{B} \theta .00 \mathrm{n} \theta .0$ & 0.00 & 0.0 & 0.18 & 0.49 & 0.78 & 21.98 & 8.22 & -2.31 & $0.00 / 13.8$ \\
\hline BQ.05n2.9 & 0.05 & -2.9 & 0.20 & 0.52 & 0.78 & 21.97 & 8.36 & -2.20 & $0.00 / 13.8$ \\
\hline $\mathrm{B} 0.05 \mathrm{n} 2.7$ & & -2.7 & 0.07 & 0.18 & 0.78 & 22.05 & 8.73 & -2.42 & $0.00 / 13.8$ \\
\hline BO. $05 \mathrm{n} 2.4$ & & -2.4 & 0.26 & 0.72 & 0.87 & 22.78 & 13.17 & -2.09 & $0.00 / 13.8$ \\
\hline B $0.05 n 2.1$ & & -2.1 & 4.52 & 8.39 & 0.84 & 22.53 & 12.94 & -1.86 & $0.67 / 7.5$ \\
\hline BQ.10n2.9 & 0.10 & -2.9 & 0.27 & 0.71 & 0.81 & 22.31 & 8.33 & -2.20 & $0.00 / 13.8$ \\
\hline BO. $20 \mathrm{n} 2.9$ & 0.20 & & 0.71 & 1.95 & 0.79 & 22.10 & 10.43 & -2.09 & $0.00 / 13.8$ \\
\hline BO. $50 \mathrm{n} 2.9$ & 0.50 & & 19.26 & 20.80 & 1.08 & 24.49 & 15.42 & -1.19 & $1.13 / 5.4$ \\
\hline \multicolumn{10}{|c|}{ h111 } \\
\hline $\mathrm{B} 0.00 \mathrm{n} 0.0$ & 0.00 & 0.0 & 0.15 & 0.36 & 1.06 & 24.40 & 10.71 & -2.65 & $0.00 / 13.8$ \\
\hline BQ.05n2.9 & 0.05 & -2.9 & 0.18 & 0.45 & 1.05 & 24.26 & 9.13 & -2.54 & $0.00 / 13.8$ \\
\hline $\mathrm{B} 0.05 \mathrm{n} 2.7$ & & -2.7 & 0.10 & 0.23 & 1.07 & 24.43 & 11.99 & -2.65 & $0.00 / 13.8$ \\
\hline BO. $05 \mathrm{n} 2.4$ & & -2.4 & 0.34 & 0.91 & 0.93 & 23.29 & 13.26 & -2.31 & $0.00 / 13.8$ \\
\hline BO.05n2.1 & & -2.1 & 3.13 & 8.98 & 0.88 & 22.92 & 16.41 & -1.98 & $0.00 / 13.8$ \\
\hline BO.10n2.9 & 0.10 & -2.9 & 0.26 & 0.64 & 1.06 & 24.38 & 10.15 & -2.20 & $0.00 / 13.8$ \\
\hline Bo.20n2.9 & 0.20 & & 0.83 & 2.21 & 1.19 & 25.32 & 11.94 & -2.20 & $0.00 / 13.8$ \\
\hline B0.50n2.9 & 0.50 & & 32.33 & 65.88 & 1.95 & 29.86 & 18.98 & -0.40 & $0.00 / 13.8$ \\
\hline
\end{tabular}

Notes. For each galaxy we computed the $V$-band total stellar luminosity $L_{\mathrm{V}}$, the stellar mass $M_{\star}$, the virial mass $M_{200}$, the virial radius $R_{200}$, the stellar line-of-sight velocity dispersion $\sigma_{\mathrm{LOS}}$, and the mode of the stellar metallicity distribution function $[\mathrm{Fe} / \mathrm{H}]$, all defined inside one virial radius. In the first row different PMF models are shown at a variety of magnetic spectral indices between -2.9 to -2.1 for constant $B_{\lambda}=0.05$, and in the second row for a constant spectral index at $n_{B}=-2.9$ and magnetic field strengths in the range $B_{\lambda}=0.05-0.50 \mathrm{nG}$. ${ }^{(a)} \mathrm{Halos}$ that did not reach $z=0$ crashed at the redshift specified here. The reason some simulations crashed at $z>0$ is due to the high gas density coupled to a high stellar mass and strong stellar feedback. The simulation time steps in dense regions is dominated by the Courant condition $\Delta t \sim \Delta x / c_{\mathrm{s}}$ with $c_{\mathrm{s}}$ the sound speed and $\Delta x$ the spatial resolution. As $\Delta x \sim 1 / \rho^{1 / 3}$, the time steps shrink with increasing density. In the presence of stellar feedback, which heats up the gas in dense regions and subsequently increases the sound speed, the time step shrinkage becomes worse. As the current version of our code uses a limit on the shortest time steps, simulations crash in extreme conditions (hot-dense gas). The problem mainly occurs for the most massive halos where the gas density is higher, triggering a stronger cooling and higher star formation, which leads to a significant gas heating. By removing the time step limit, we can avoid the simulation crash, however at the expense of running simulations for many more CPU-hours. 
Table A.1. continued.

\begin{tabular}{|c|c|c|c|c|c|c|c|c|c|}
\hline $\begin{array}{l}\text { Model } \\
\text { ID }\end{array}$ & $\begin{array}{c}B_{\lambda} \\
{[\mathrm{nG}]}\end{array}$ & $\begin{array}{c}n_{B} \\
- \\
\end{array}$ & $\begin{array}{c}L_{\mathrm{V}} \\
{\left[10^{6} L_{\odot}\right]}\end{array}$ & $\begin{array}{c}M_{\star} \\
{\left[10^{6} M_{\odot}\right]}\end{array}$ & $\begin{array}{c}M_{200} \\
{\left[10^{9} M_{\odot}\right]}\end{array}$ & $\begin{array}{l}R_{200} \\
\mathrm{kpc}\end{array}$ & $\begin{array}{c}\sigma_{\mathrm{LOS}} \\
{\left[\mathrm{kms}^{-1}\right]}\end{array}$ & $\begin{array}{c}{[\mathrm{Fe} / \mathrm{H}]} \\
\operatorname{dex}\end{array}$ & $\begin{array}{r}\text { Redshift/ Time } \\
-/[\mathrm{Gyr}]\end{array}$ \\
\hline \multicolumn{10}{|c|}{ h122 } \\
\hline $\mathrm{B} \theta .00 \mathrm{n} \theta .0$ & 0.00 & 0.0 & 0.11 & 0.26 & 0.93 & 23.37 & 9.02 & -2.65 & $0.00 / 13.8$ \\
\hline BQ.05n2.9 & 0.05 & -2.9 & 0.12 & 0.30 & 0.96 & 23.61 & 9.22 & -2.65 & $0.00 / 13.8$ \\
\hline BQ $.05 \mathrm{n} 2.7$ & & -2.7 & 0.05 & 0.12 & 0.89 & 22.98 & 10.53 & -3.10 & $0.00 / 13.8$ \\
\hline BQ.05n2. 4 & & -2.4 & 0.12 & 0.32 & 0.86 & 22.77 & 13.81 & -2.42 & $0.00 / 13.8$ \\
\hline $\mathrm{B} 0.05 \mathrm{n} 2.1$ & & -2.1 & 1.48 & 4.13 & 0.84 & 22.56 & 15.05 & -1.98 & $0.00 / 13.8$ \\
\hline BQ.10n2.9 & 0.10 & -2.9 & 0.18 & 0.46 & 0.91 & 23.17 & 8.77 & -2.09 & $0.00 / 13.8$ \\
\hline BO. $20 \mathrm{n} 2.9$ & 0.20 & & 0.52 & 1.36 & 0.90 & 23.12 & 10.92 & -2.20 & $0.00 / 13.8$ \\
\hline $\mathrm{B} 0.50 \mathrm{n} 2.9$ & 0.50 & & 25.93 & 53.81 & 1.54 & 27.60 & 18.33 & -0.40 & $0.00 / 13.8$ \\
\hline \multicolumn{10}{|c|}{ h159 } \\
\hline $\mathrm{B} \theta .00 \mathrm{n} \theta .0$ & 0.00 & 0.0 & 0.40 & 1.03 & 0.68 & 21.02 & 9.31 & -2.42 & $0.00 / 13.8$ \\
\hline BQ.05n2.9 & 0.05 & -2.9 & 0.43 & 1.09 & 0.68 & 21.05 & 8.96 & -1.75 & $0.00 / 13.8$ \\
\hline BQ.05n2.7 & & -2.7 & 0.27 & 0.69 & 0.67 & 20.93 & 10.23 & -2.42 & $0.00 / 13.8$ \\
\hline BQ. $05 \mathrm{n} 2.4$ & & -2.4 & 0.40 & 1.10 & 0.59 & 20.09 & 11.59 & -2.20 & $0.00 / 13.8$ \\
\hline $\mathrm{B} 0.05 \mathrm{n} 2.1$ & & -2.1 & 2.08 & 6.06 & 0.65 & 20.73 & 15.12 & -1.86 & $0.00 / 13.8$ \\
\hline BQ.10n2.9 & 0.10 & -2.9 & 0.50 & 1.29 & 0.68 & 21.00 & 9.98 & -1.75 & $0.00 / 13.8$ \\
\hline $\mathrm{B} 0.20 \mathrm{n} 2.9$ & 0.20 & & 0.91 & 2.49 & 0.67 & 20.93 & 10.49 & -2.20 & $0.00 / 13.8$ \\
\hline BQ. $50 \mathrm{n} 2.9$ & 0.50 & & 15.10 & 36.82 & 1.64 & 28.18 & 18.10 & -1.19 & $0.00 / 13.8$ \\
\hline \multicolumn{10}{|c|}{ h168 } \\
\hline $\mathrm{BO} .00 \mathrm{n} \theta .0$ & 0.00 & 0.0 & 0.11 & 0.27 & 0.54 & 19.42 & 8.68 & -2.76 & $0.00 / 13.8$ \\
\hline BQ.05n2.9 & 0.05 & -2.9 & 0.66 & 1.79 & 2.88 & 34.01 & 9.60 & -2.20 & $0.00 / 13.8$ \\
\hline $\mathrm{B} 0.05 \mathrm{n} 2.7$ & & -2.7 & 0.31 & 0.81 & 2.70 & 33.29 & 10.70 & -2.31 & $0.00 / 13.8$ \\
\hline BQ. $05 \mathrm{n} 2.4$ & & -2.4 & 0.32 & 0.88 & 1.14 & 24.93 & 11.90 & -2.20 & $0.00 / 13.8$ \\
\hline $\mathrm{B} 0.05 \mathrm{n} 2.1$ & & -2.1 & 1.51 & 4.43 & 0.52 & 19.18 & 11.08 & -1.98 & $0.00 / 13.8$ \\
\hline BQ.10n2.9 & 0.10 & -2.9 & 0.70 & 1.92 & 2.68 & 33.20 & 10.15 & -2.20 & $0.00 / 13.8$ \\
\hline $\mathrm{B} 0.20 \mathrm{n} 2.9$ & 0.20 & & 1.31 & 3.67 & 2.31 & 31.61 & 12.15 & -1.41 & $0.00 / 13.8$ \\
\hline BQ. $50 \mathrm{n} 2.9$ & 0.50 & & 18.36 & 40.09 & 1.43 & 26.94 & 18.34 & -1.08 & $0.00 / 13.8$ \\
\hline \multicolumn{10}{|c|}{ h177 } \\
\hline BQ. $00 \mathrm{n} \theta .0$ & 0.00 & 0.0 & 0.18 & 0.47 & 0.52 & 19.26 & 8.35 & -2.31 & $0.00 / 13.8$ \\
\hline BQ.05n2.9 & 0.05 & -2.9 & 0.19 & 0.50 & 0.52 & 19.22 & 7.53 & -2.54 & $0.00 / 13.8$ \\
\hline $\mathrm{B} 0.05 \mathrm{n} 2.7$ & & -2.7 & 0.07 & 0.17 & 0.51 & 19.07 & 8.02 & -2.65 & $0.00 / 13.8$ \\
\hline BQ. $05 \mathrm{n} 2.4$ & & -2.4 & 0.18 & 0.49 & 0.37 & 17.08 & 10.72 & -2.09 & $0.00 / 13.8$ \\
\hline $\mathrm{B} 0.05 \mathrm{n} 2.1$ & & -2.1 & 1.14 & 3.18 & 0.68 & 21.06 & 13.11 & -1.98 & $0.00 / 13.8$ \\
\hline BQ.10n2.9 & 0.10 & -2.9 & 0.25 & 0.65 & 0.54 & 19.42 & 8.27 & -2.31 & $0.00 / 13.8$ \\
\hline $\mathrm{B} 0.20 \mathrm{n} 2.9$ & 0.20 & & 0.52 & 1.40 & 0.56 & 19.67 & 8.64 & -2.20 & $0.00 / 13.8$ \\
\hline BQ. $50 \mathrm{n} 2.9$ & 0.50 & & 5.63 & 17.27 & 0.94 & 23.41 & 13.54 & -1.19 & $0.00 / 13.8$ \\
\hline
\end{tabular}


M. Sanati et al.: Dwarf galaxies as a probe of Primordial Magnetic Fields

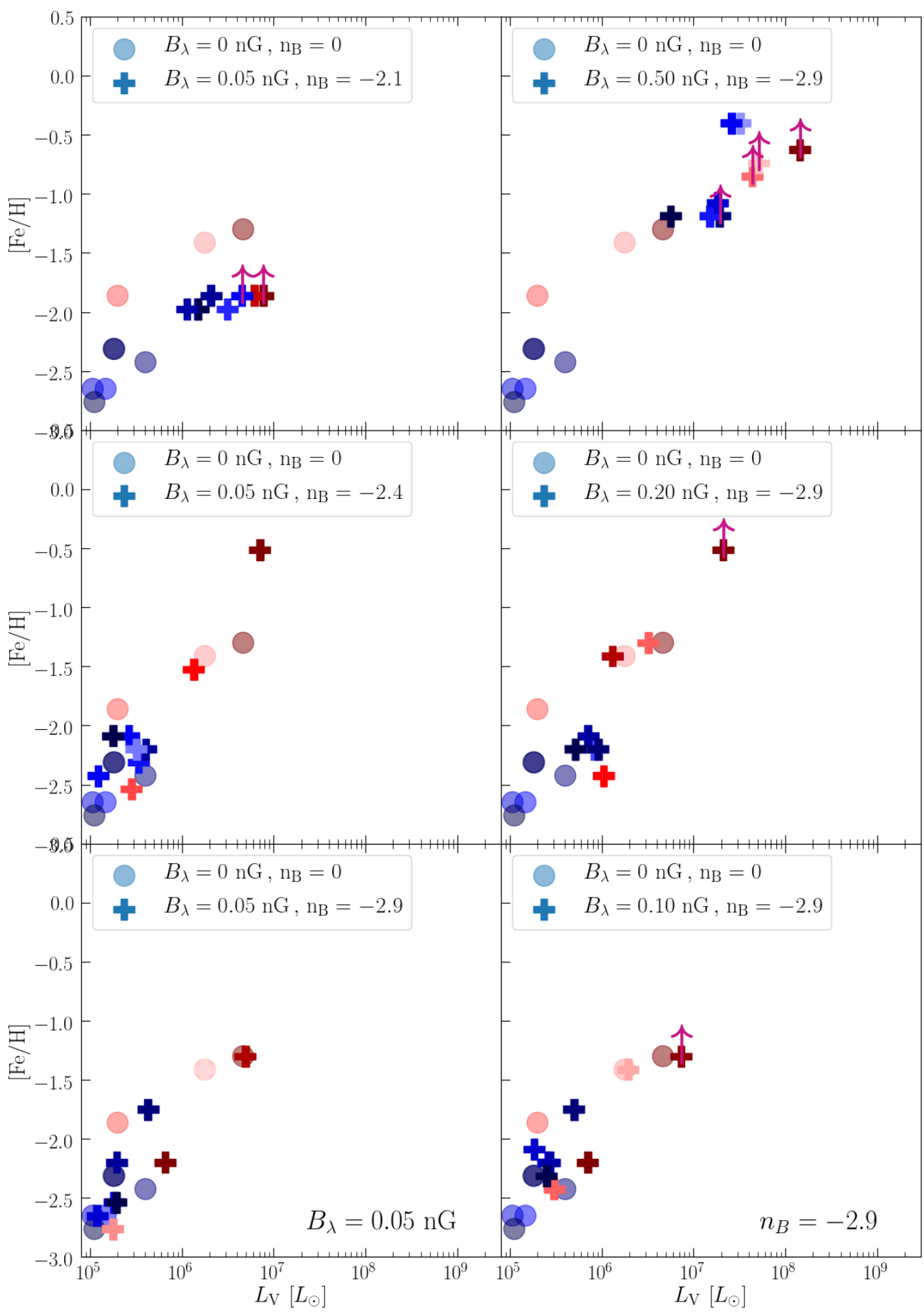

Fig. A.1. Mean metallicity versus stellar $V$-band luminosity. Each object corresponds to one model galaxy with the physical properties given in Table A.1 for which $[\mathrm{Fe} / \mathrm{H}]$ is computed as the median of the galaxy stellar metallicity distribution function. The color-coding shows the halo mass of each model galaxy. The purple arrows indicate models that did not reach $z=0$ and are expected to have a slightly higher metallicity. 


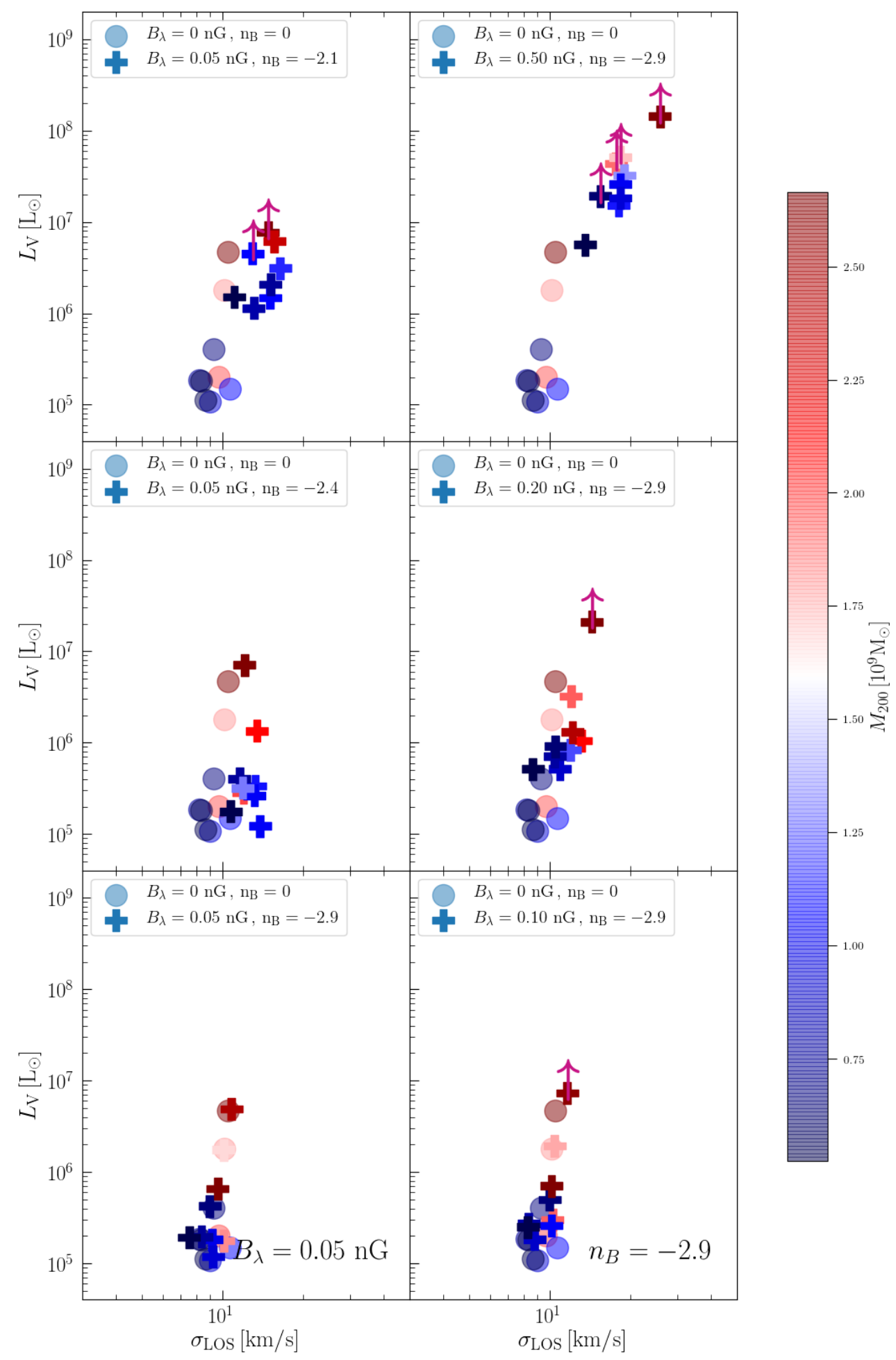

Fig. A.2. $V$-band luminosity as a function of line-of-sight velocity dispersion in each model galaxy with physical properties given in Table A.1 in each magnetic field model compared to the no-magnetic-field model. The color-coding shows the halo mass of each model galaxy. The purple arrows indicate models that did not reach $z=0$ and are expected to have a slightly brighter luminosity. 